Algebraic 85 Geometric Topology

Volume 3 (2003) 399-433

Published: 8 May 2003

ATG

\title{
Espaces profinis et problèmes de réalisabilité
}

\author{
FrançOIS-XaVier DeHON \\ GÉRALD GAUDENS
}

\begin{abstract}
The mod $p$ cohomology of a space comes with an action of the Steenrod Algebra. L. Schwartz [Sc2] proved a conjecture due to N. Kuhn $[\mathrm{Ku}]$ stating that if the mod $p$ cohomology of a space is in a finite stage of the Krull filtration of the category of unstable modules over the Steenrod algebra then it is locally finite. Nevertheless his proof involves some finiteness hypotheses. We show how one can remove those finiteness hypotheses by using the homotopy theory of profinite spaces introduced by F. Morel $[\mathrm{Mo}]$, thus obtaining a complete proof of the conjecture. For that purpose we build the Eilenberg-Moore spectral sequence and show its convergence in the profinite setting.
\end{abstract}

AMS Classification 55S10; 55T20, 57T35

Keywords Steenrod operations, nilpotent modules, realization, Eilenberg-Moore spectral sequence, profinite spaces

\section{Introduction}

La cohomologie à coefficients dans un corps fini d'un espace topologique est naturellement munie d'une structure de module instable sur l'algèbre de Steenrod [SE]. La question se pose de caractériser, parmi les modules instables, ceux qui sont isomorphes à la cohomologie d'un espace topologique. De tels modules instables sont dit topologiquement réalisables.

Déterminer si un module instable est réalisable est en général difficile. Par exemple, le fameux résultat de J.F. Adams sur les éléments de l'homotopie des sphères d'invariant de Hopf un $[\mathrm{Ad}]$ se paraphrase en la non réalisabilité de certains modules finis.

Un critère de réalisabilité simple, utilisé dans la suite, repose sur la remarque suivante : la cohomologie d'un espace est aussi munie d'une structure d'algèbre, laquelle est compatible avec l'action de l'algèbre de Steenrod [SE, Sc1]. En particulier, l'élévation au carré y est donnée par une opération de Steenrod. En 
d'autres termes, l'existence d'une structure d'algèbre compatible fournit une condition nécessaire à la réalisabilité d'un module instable.

Dans un article récent $[\mathrm{Ku}], \mathrm{N}$. Kuhn énonce, et démontre dans certains cas, des conjectures sur la structure de module instable de la cohomologie des espaces. Voici celle qu'on étudie ici.

\subsection{La conjecture de non-réalisation forte due à N. Kuhn}

Soit $p$ un nombre premier. Notons $\mathcal{U}$ la catégorie des modules instables sur l'algèbre de Steenrod modulo $p$. Soit $\mathcal{U}_{n}$ la sous-catégorie pleine de $\mathcal{U}$ dont les objets sont ceux annulés par $\overline{\mathrm{T}}^{n}$, le foncteur de Lannes réduit itéré $n$ fois (voir la partie 5). La suite $\left(\mathcal{U}_{n}\right)$ coïncide avec la filtration de Gabriel-Krull de la catégorie $\mathcal{U}$ (voir la partie 6 ).

Le premier cran $\mathcal{U}_{0}$ de cette filtration est la sous-catégorie pleine de $\mathcal{U}$ dont les objets sont les modules localement finis, i.e. les modules $M$ tels que pour tout élément $x$ de $M$, il existe un entier $\ell$ tel que toute opération de degré supérieur à $\ell$ opère trivialement sur $x$.

N. Kuhn a proposé la conjecture suivante $[\mathrm{Ku}$, strong realization conjecture, p.324] :

Conjecture de non-réalisation forte $[\mathbf{K u}]$ Soit $X$ un espace topologique. Si la cohomologie modulo $p$ de $X$ est dans $\mathcal{U}_{n}$ pour un certain entier $n$, alors elle est localement finie.

\subsection{L'approche de L. Schwartz}

L. Schwartz a démontré cette conjecture pour $p=2$ sous certaines restrictions de finitude. Précisément, il a démontré [Sc3, Théorème 0.1] :

Théorème 1.1 [Sc3] Soit $X$ un espace, et soit $M$ sa cohomologie modulo 2. Supposons que $M$ est dans $\mathcal{U}_{n}$ pour un certain entier $n$, et que pour tout entier $s$ le quotient $M_{s} / M_{s+1}$ a un nombre fini de générateurs sur l'algèbre de Steenrod. Alors $M$ est localement fini, c'est-à-dire $M \in \mathcal{U}_{0}$.

Dans l'énoncé, $M_{s}$ désigne le sous-module instable de $M$ formé des éléments $s$-nilpotents (voir la partie 6). 
Rappelons la trame de la démonstration de L. Schwartz. On procède par l'absurde en supposant qu'il existe un espace $X$ dont la cohomologie modulo 2 est dans un cran fini $\mathcal{U}_{n}$ de la filtration de Krull sans être localement finie. On aboutit à une contradiction en deux étapes:

La première étape est la réduction de $N$. Kuhn : On montre qu'il existe alors un (nouvel) espace $X$ dont la cohomologie est dans le premier cran $\mathcal{U}_{1}$ de la filtration de Krull sans être localement finie.

La seconde étape repose sur l'utilisation de la suite spectrale d'Eilenberg-Moore associée à la fibration des chemins de $X$. On précise alors la structure particulière des modules qui sont dans le premier cran de la filtration de Krull sans être localement finis pour construire pour un certain entier $d$ et pour tout entier $i$ assez grand des classes $\alpha_{i, d}$ dans la cohomologie de $X$, qui vérifient les deux propriétés suivantes :

- Le cup carré de $\alpha_{i, d}$ est nul pour tout entier $i$ assez grand,

- La classe $\alpha_{i, d}$ est exactement $d$-nilpotente pour tout entier $i$ assez grand.

Les propriétés particulières de ces classes $\alpha_{i, d}$ montrent qu'elles induisent dans la cohomologie de l'espace de lacets $\Omega X$, à travers l'homomorphisme de coin, des classes $\alpha_{i, d-1}$, qui vérifient :

- Le cup carré de la classe $\alpha_{i, d-1}$ est nul pour tout entier $i$ assez grand,

- La classe $\alpha_{i, d-1}$ est exactement $(d-1)$-nilpotente.

Par une récurrence descendante, on arrive à la contradiction qu'il existe des classes $\alpha_{i, 0}$ dans $\mathrm{H}^{*} \Omega^{d} X$ qui, pour $i$ assez grand, sont simultanément 0nilpotentes (i.e. réduites au sens de la structure d'algèbre de la cohomologie) et de cup-carré nul. Ceci contredit l'existence d'une structure d'algèbre compatible avec la structure de module sur l'algèbre de Steenrod sur $\mathrm{H}^{*} \Omega^{d} X$.

L'utilisation de l'interprétation géométrique du foncteur $\mathrm{T}$ et de la suite spectrale d'Eilenberg-Moore dans la catégorie des espaces impose les hypothèses de finitude faites dans le théorème 1.1. Cet article expose le moyen de contourner ces hypothèses en utilisant la théorie de l'homotopie des espaces profinis.

\subsection{De l'utilisation des espaces profinis dans les problèmes de non réalisabilité}

Le premier point est qu'on connait une interprétation géométrique inconditionnelle du foncteur de Lannes [Mo], le prix à payer étant d'échanger la catégorie des espaces par celle des espaces profinis. 
Le second point est que nous construisons dans cet article une suite spectrale d'Eilenberg-Moore dans le cadre des espaces profinis. Il faut pour cela donner un sens à la notion d'espace de lacets d'un espace profini pointé. Le formalisme adéquat pour développer ces deux points est l'algèbre homotopique.

L'approche utilisée par L. Schwartz s'applique alors mutatis mutandi et permet de montrer le théorème suivant.

Théorème 1.2 Soit $X$ un espace profini. Si la cohomologie modulo 2 continue de $X$ est dans un cran fini de la filtration de Krull, alors elle est localement finie.

On sait que la cohomologie d'un espace coïncide avec la cohomologie continue de son complété profini (voir la section 2). Par conséquent, en corollaire du théorème 1.2 , on établit la conjecture de non réalisation forte pour $p=2$.

Théorème 1.3 Soit $X$ un espace. Si la cohomologie modulo 2 de $X$ est dans un cran fini de la filtration de Krull, alors elle est localement finie.

\subsection{Organisation de l'article}

L'article est organisé de la manière suivante. Après avoir rappelé les points de la théorie homotopique des espaces profinis que nous utilisons, nous détaillons les constructions des sommes amalgamées homotopiques et produits fibrés homotopiques, et en particulier des espaces de lacets. Nous construisons alors la suite spectrale d'Eilenberg-Moore d'un produit fibré homotopique et en explicitons la structure algébrique. Suivent quelques rappels sur la théorie de Lannes dans le cadre des espaces profinis. Dans la dernière section, on expose, suivant la méthode de L. Schwartz, la démonstration du Théorème 1.2, ce qui établit la conjecture de non réalisation forte (Théorème 1.3) pour $p=2$. Dans un appendice, on détaille certains points techniques.

La preuve du théorème 1.2 est, tant dans sa ligne que dans son aspect technique, très semblable à celle de L. Schwartz [Sc3]. Néanmoins, dans un soucis de clarté, nous avons donné dans cet article une démonstration aussi complète que possible. Une des raisons qui motivent ce choix est qu'il nous faut nous soustraire des hypothèses de finitude faites dans [Sc3]. En effet, on y trouve [Sc3, p. 523]: 'On fera partout l'hypothèse que les modules instables considérés sont de dimension finie en chaque degré.' De plus, [Sc3, lemme 1.12, p. 530] n'est pas vrai sans hypothèses de finitude, ce qui nous empêche de nous appuyer sur 
[Sc3, propositions 1.9 et 1.10]. Malheureusement, ces propositions sont utilisées en divers points cruciaux de l'article, par exemple dans la démonstration de [Sc3, proposition 3.2] ou encore dans le calcul final [Sc3, p. 342].

Remerciements Le second auteur remercie chaleureusement le Centre de Recerca Matemàtica de Barcelone ainsi que le laboratoire Jean-Alexandre Dieudonné (Université de Nice) pour leur accueil durant la préparation de ce travail.

Nous remercions vivement Lionel Schwartz pour l'intérêt qu'il a pris pour notre travail.

\section{Théorie homotopique des espaces profinis (d'après $[\mathrm{Mo}])$}

\section{$2.1 \quad$ Espaces profinis}

Une catégorie $\mathcal{I}$ est dite petite si la collection de ses objets forme un ensemble. Elle est dite filtrante si :

(i) pour toute paire d'objets $i, j$ de $\mathcal{I}$, il existe un objet $k$ et des morphismes $k \rightarrow i$ et $k \rightarrow j$.

(ii) pour toute paire de morphismes $i \rightrightarrows j$ entre deux objets $i, j$ il existe un objet $k$ et un morphisme $k \rightarrow i$ tel que les composées $k \rightarrow i \rightrightarrows j$ soient égales.

Soit $\mathcal{C}$ une catégorie. Un pro-objet de $\mathcal{C}$ est la donnée d'une petite catégorie filtrante $\mathcal{I}$ et d'un foncteur de $\mathcal{I}$ dans $\mathcal{C}$. On dit aussi un diagramme filtrant d'objets de $\mathcal{C}$. Un morphisme entre deux pro-objets $X(-): \mathcal{I} \rightarrow \mathcal{C}$ et $Y(-): \mathcal{J} \rightarrow \mathcal{C}$ est un élément de l'ensemble $\lim _{i} \operatorname{colim}_{j} \operatorname{Hom}(X(j), Y(i))$. Les pro-objets et leurs morphismes forment une catégorie notée pro- $\mathcal{C}$. (Voir par exemple [AM, appendice].)

Un ensemble profini est un espace topologique compact totalement discontinu. La limite dans $\mathcal{T}_{o p}$ d'un diagramme filtrant d'ensembles finis (discrets) est un ensemble profini. Inversement tout ensemble profini apparaît canoniquement comme la limite filtrante de ses quotients finis $X / R, R$ décrivant les relations d'équivalence ouvertes sur $X$. (Voir par exemple [Do, 2.8].)

Notre catégorie d'espaces est celle des ensembles simpliciaux, qu'on note $\mathcal{S}$ (voir par exemple [BK, chap. VIII]). 
On appelle espace profini un objet simplicial dans la catégorie des ensembles profinis. Les espaces profinis et leurs morphismes forment une catégorie notée $\widehat{\mathcal{S}}$.

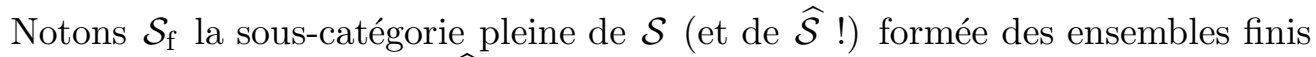
simpliciaux. Le foncteur $\widehat{\mathcal{S}} \rightarrow$ pro- $\mathcal{S}_{\mathrm{f}}$ qui associe à un espace profini $X$ le diagramme filtrant de ses quotients finis simpliciaux $X / R$ est une équivalence de catégories d'inverse le foncteur limite pro- $\mathcal{S}_{\mathrm{f}} \rightarrow \widehat{\mathcal{S}}(\mathrm{cf}[\mathrm{Q} 2$, lemma 2.3]).

\subsection{Théorie homotopique}

Soit $X$ un espace profini. On définit la cohomologie modulo $p$ continue de $X$, notée $\mathrm{H}^{*} X$, comme l'homologie du complexe des cochaînes continues de $X$ à valeurs dans le groupe discret $\mathbb{Z} / p$. Cette cohomologie est aussi la colimite filtrante des cohomologies modulo $p$ ordinaires des quotients finis simpliciaux de $X$. Elle hérite en particulier de la structure que possède la cohomologie modulo $p$ ordinaire d'un espace : c'est une algèbre instable sur l'algèbre de Steenrod (voir par exemple [Sc1]).

La catégorie $\widehat{\mathcal{S}}$ possède une structure de catégorie de modèles fermée avec pour équivalences faibles les morphismes induisant un isomorphisme en cohomologie modulo $p$ continue et pour cofibrations les monomorphismes [Mo, théorème 1].

On en déduit immédiatement une structure de catégorie de modèles sur la catégorie pointée $\widehat{\mathcal{S}}_{\mathrm{pt}}$ formée des espaces profinis munis d'un point base. Observons que l'oubli du point base $\widehat{\mathcal{S}}_{\mathrm{pt}} \rightarrow \widehat{\mathcal{S}}$ admet un adjoint à gauche : le foncteur qui associe à un espace profini $X$ la réunion disjointe de $X$ et d'un point base, qu'on note $X_{+}$.

\subsection{Complétion profinie}

Le foncteur d'oubli de la topologie $\widehat{\mathcal{S}} \rightarrow \mathcal{S}$ admet un adjoint à gauche : le foncteur de complétion profinie qui associe à un ensemble simplicial $X$ la limite dans $\widehat{\mathcal{S}}$ de ses quotients finis simpliciaux $X / R$. Par construction, les morphismes $X \rightarrow X / R$ induisent un isomorphisme de la cohomologie modulo $p$ continue du complété profini de $X$ dans la cohomologie modulo $p$ ordinaire de $X$. 


\subsection{Pro- $p$-complétion et résolution fibrante}

Soit $X$ un ensemble simplicial. On note $\operatorname{Res}^{\bullet}(X)$ sa $\mathbb{F}_{p}$-résolution cosimpliciale $\left[\mathrm{BK}\right.$, part I], $\operatorname{Tot}_{s} \operatorname{Res}^{\bullet}(X)$ le $s$-ième espace total partiel associé, et $\mathrm{P}^{t} \operatorname{Tot}_{s} \operatorname{Res}^{\bullet}(X)$ la $t$-ième troncature de Postnikov de l'espace $\operatorname{Tot}_{s} \operatorname{Res}^{\bullet}(X)$. Alors :

- L'espace $\operatorname{Tot}_{0} \operatorname{Res}^{\bullet}(X)$ est un $\mathbb{F}_{p}$-espace affine simplicial et les morphismes $\operatorname{Tot}_{s+1} \operatorname{Res}^{\bullet}(X) \rightarrow \operatorname{Tot}_{s} \operatorname{Res}^{\bullet}(X)$ sont des fibration principales sous l'action de $\mathbb{F}_{p}$-espaces vectoriels simpliciaux.

- Lorsque $X$ est un ensemble fini simplicial, les espaces $\operatorname{Tot}_{s} \operatorname{Res}{ }^{\bullet}(X)$ le sont également.

- Lorsque $X$ est connexe, les morphismes naturels $X \rightarrow \operatorname{Tot}_{s} \operatorname{Res}^{\bullet}(X)$ induisent un isomorphisme $\operatorname{colim}_{s} \mathrm{H}^{*} \operatorname{Tot}_{s} \operatorname{Res}^{\bullet}(X) \rightarrow \mathrm{H}^{*} X$ [BK, chap. III, $\S 6]$.

On déduit des deux premiers points que lorsque $X$ est un ensemble fini simplicial, les espaces $\mathrm{P}^{t} \operatorname{Tot}_{s} \operatorname{Res}^{\bullet}(X)$ sont des espaces $p$-finis ; c'est à dire ils sont finis simpliciaux, fibrants dans $\mathcal{S}$, ont un nombre fini de composantes connexes et les groupes d'homotopie de chacune d'entre elles sont des $p$-groupes finis, triviaux sauf pour un nombre fini d'entre eux.

On déduit du troisième point que les morphismes naturels $X \rightarrow \mathrm{P}^{t} \operatorname{Tot}_{s} \operatorname{Res}^{\bullet}(X)$ induisent un isomorphisme $\operatorname{colim}_{s, t} \mathrm{H}^{*} \mathrm{P}^{t} \operatorname{Tot}_{s} \operatorname{Res}^{\bullet}(X) \rightarrow \mathrm{H}^{*} X$ lorsque $X$ est fini simplicial.

Supposons maintenant que $X$ est un espace profini. On note $\operatorname{Tot}_{s} X$ l'espace profini $\lim _{\mathrm{R}} \operatorname{Tot}_{s} \operatorname{Res}^{\bullet}(X / R)$. Alors $\operatorname{Tot}_{0} X$ est un $\mathbb{F}_{p}$-espace affine profini simplicial et les morphismes

$$
\operatorname{Tot}_{s+1} X \rightarrow \operatorname{Tot}_{s} X
$$

sont des fibrations principales sous l'action de $\mathbb{F}_{p}$-espaces vectoriels profinis simpliciaux. En particulier les morphismes $\operatorname{Tot}_{0} X \rightarrow$ pt et $\operatorname{Tot}_{s+1} X \rightarrow \operatorname{Tot}_{s} X$ sont des fibrations dans $\widehat{\mathcal{S}}$ (voir [Mo, 2.1]).

On note $\widehat{X}(-)$ le diagramme filtrant formé des espaces $p$-finis $\mathrm{P}^{t} \operatorname{Tot}_{s} \operatorname{Res}{ }^{\bullet}(X / R), \quad t$ et $s$ décrivant l'ensemble des entiers positifs et $R$ l'ensemble des relations d'équivalence simpliciales ouvertes de $X$, et $\mathrm{R} X$ la limite de $\widehat{X}(-)$ dans $\widehat{\mathcal{S}}$. Le morphisme canonique $X \rightarrow \mathrm{R} X$ est une équivalence faible faisant de $\mathrm{R} X$ une résolution fibrante fonctorielle de $X$. On appelle le diagramme $\widehat{X}(-)$ le pro- $p$-complété de $X$. 


\subsection{Objets fonctionnels}

Soient $X$ et $Y$ deux espaces profinis pointés. On note $X \vee Y$ leur somme et $X \wedge Y$ le quotient $(X \times Y) /(X \vee Y)$. L'espace profini $X \wedge Y$ est fonctoriel en $X$ et $Y$.

Notons, pour $W$ un espace profini pointé, $\overline{\mathrm{H}}^{*} W$ sa cohomologie modulo $p$ continue réduite, noyau du morphisme $\mathrm{H}^{*} W \rightarrow \mathrm{H}^{*}$ pt. On dispose d'un morphisme canonique $\overline{\mathrm{H}}^{*} X \otimes \overline{\mathrm{H}}^{*} Y \rightarrow \overline{\mathrm{H}}^{*}(X \wedge Y)$ qui est un isomorphisme si $X$ et $Y$ sont des ensembles finis simpliciaux donc un isomorphisme en général par commutation des colimites filtrantes aux produits tensoriels.

Il existe un ensemble simplicial pointé $\operatorname{hom}_{\mathrm{pt}}(X, Y)$ caractérisé par la bijection

$$
\operatorname{Hom}_{\mathcal{S}_{\mathrm{pt}}}\left(W, \operatorname{hom}_{\mathrm{pt}}(X, Y)\right) \simeq \operatorname{Hom}_{\widehat{\mathcal{S}}_{\mathrm{pt}}}(X \wedge W, Y)
$$

naturelle en $W \in\left(\mathcal{S}_{\mathrm{f}}\right)_{\mathrm{pt}}$, et faisant de $\widehat{\mathcal{S}}_{\mathrm{pt}}$ une catégorie de modèles fermée simpliciale (cf $[\mathrm{Q}, \mathrm{II}, \S 1,2]$ ). Le fait que pour toute cofibration $A \rightarrow B$ et pour toute fibration $X \rightarrow Y$ dans $\widehat{\mathcal{S}}_{\mathrm{pt}}$ le morphisme

$$
\operatorname{hom}_{\mathrm{pt}}(B, X) \rightarrow \operatorname{hom}_{\mathrm{pt}}(A, X) \times_{\operatorname{hom}_{\mathrm{pt}}(A, Y)} \operatorname{hom}_{\mathrm{pt}}(B, Y)
$$

est une fibration de $\mathcal{S}_{\mathrm{pt}}$ et une équivalence faible si de plus $A \rightarrow B$ ou $X \rightarrow Y$ est une équivalence faible dans $\widehat{\mathcal{S}}_{\mathrm{pt}}$ vient par adjonction des deux propriétés suivantes :

- Une équivalence faible dans $\mathcal{S}_{\mathrm{pt}}$ entre ensembles finis simpliciaux pointés est une équivalence faible dans $\widehat{\mathcal{S}}_{\mathrm{pt}}$.

- Pour tout monomorphisme $W \rightarrow W^{\prime}$ entre ensembles finis simpliciaux pointés (plus généralement pour toute cofibration $W \rightarrow W^{\prime}$ dans $\widehat{\mathcal{S}}_{\mathrm{pt}}$ ) le morphisme

$$
\left(A \wedge W^{\prime}\right) \cup_{A \wedge W}(B \wedge W) \rightarrow B \wedge W^{\prime}
$$

est une cofibration de $\widehat{\mathcal{S}}_{\text {pt }}$ qui est une équivalence faible si $W \rightarrow W^{\prime}$ ou $A \rightarrow B$ est une équivalence faible.

Supposons maintenant $X$ fini simplicial et notons $\operatorname{Sk}_{n} X$ son $n$-ième squelette. L'ensemble simplicial $\mathbf{h o m}_{\mathrm{pt}}(X, Y)$ est alors naturellement profini car limite filtrante des ensembles finis simpliciaux $\mathbf{h o m}_{\mathrm{pt}}\left(\mathrm{Sk}_{n} X, Y / R\right)$, et comme tel l'adjoint à droite en $Y$ du foncteur $Z \mapsto X \wedge Z, \widehat{\mathcal{S}}_{\text {pt }} \rightarrow \widehat{\mathcal{S}}_{\text {pt }}$.

La même discussion vaut dans $\widehat{\mathcal{S}}$ en remplaçant $X \wedge Y$ par $X \times Y$. Observons que si $Y$ est pointé l'ensemble simplicial $\operatorname{hom}(X, Y)$ est naturellement pointé par le morphisme constant $X \rightarrow \mathrm{pt} \rightarrow Y$ et s'identifie comme tel à l'ensemble simplicial pointé $\operatorname{hom}_{\mathrm{pt}}\left(X_{+}, Y\right)$. 
Cas particulier Notons $\mathrm{S}^{1}$ le quotient du simplexe standard de dimension 1 de $\mathcal{S}$ par son 0 -squelette. L'espace $\mathrm{S}^{1}$ est un ensemble fini simplicial pointé. A tout espace profini pointé $X$ on associe sa suspension $\Sigma X=\mathrm{S}^{1} \wedge X$ et son espace de lacets $\Omega X=\mathbf{h o m}_{\mathrm{pt}}\left(\mathrm{S}^{1}, X\right)$.

Nous terminons par les trois remarques suivantes:

- Soient $Y$ un espace profini pointé, $X$ un ensemble simplicial pointé quelconque et notons $\left(X_{\alpha}\right)$ le diagramme filtrant formé des sous-ensembles finis simpliciaux de $X$. On définit alors l'espace profini fonctionnel $\mathbf{h o m}_{\mathrm{pt}}(X, Y)$ comme la limite des espaces profinis $\mathbf{h o m}_{\mathrm{pt}}\left(X_{\alpha}, Y\right)$. Il est l'adjoint à droite en $Y$ du foncteur $\widehat{\mathcal{S}}_{\mathrm{pt}} \rightarrow \widehat{\mathcal{S}}_{\mathrm{pt}}, Z \mapsto \operatorname{colim}_{\alpha}\left(X_{\alpha} \wedge Z\right)$.

- Soient $X$ un ensemble simplicial pointé ayant un nombre fini de simplexes non dégénérés et $Y$ un espace $p$-fini pointé, alors l'espace $\mathbf{h o m}_{\mathrm{pt}}(X, Y)$ est également $p$-fini.

- Soient $Y \rightarrow Y^{\prime}$ une équivalence faible entre espaces profinis pointés fibrants et $X$ un ensemble simplicial (respectivement un espace profini) pointé, alors le morphisme $\operatorname{hom}_{\mathrm{pt}}(X, Y) \rightarrow \operatorname{hom}_{\mathrm{pt}}\left(X, Y^{\prime}\right)$ est une équivalence faible entre espaces profinis (respectivement entre ensembles simpliciaux), ceci parce qu'une équivalence faible entre espaces profinis (pointés) fibrants est une équivalence d'homotopie simpliciale [Mo, 1.4, Lemme 3]. (Nous rappelons ci-dessous la notion d'homotopie simpliciale.)

\section{Sommes amalgamées et produits fibrés homo- topiques}

\subsection{Sommes amalgamées homotopiques}

Notons $\triangle[s]$ le simplexe standard de dimension $s$ de $\mathcal{S}$; c'est un ensemble fini simplicial non fibrant dans $\mathcal{S}$ si $s$ est non nul, faiblement équivalent au point.

Les morphismes $\mathrm{d}^{0}, \mathrm{~d}^{1}: \triangle[0] \rightarrow \triangle[1]$ et $\mathrm{s}^{0}: \triangle[1] \rightarrow \triangle[0]$ induisent pour tout espace profini pointé $X$ des morphismes $X \vee X \rightarrow X \wedge \triangle[1]_{+}$et $X \wedge \triangle[1]_{+} \rightarrow X$ qui font de $X \wedge \triangle[1]_{+}$un (bon) objet cylindre pour $X$ dans $\widehat{\mathcal{S}}_{\mathrm{pt}}$, fonctoriel en $X$. (Voir par exemple $[\mathrm{DwS}]$ pour cette notion).

Notons $\{0\}$ et $\{1\}$ les images respectives de $\mathrm{d}^{1}$ et $\mathrm{d}^{0}: \triangle[0] \rightarrow \triangle[1]$. Tout morphisme $X \rightarrow Y$ entre espaces profinis pointés s'écrit comme la composée des morphismes évidents $X \wedge\{0\}_{+} \rightarrow\left(X \wedge \triangle[1]_{+}\right) \cup_{X \wedge\{1\}_{+}} Y$ et $\left(X \wedge \triangle[1]_{+}\right) \cup_{X \wedge\{1\}_{+}}$ $Y \rightarrow Y$. 
Proposition 3.1 Soit $X \rightarrow Y$ un morphisme entre espaces profinis pointés ; alors :

- Le morphisme $X \wedge\{0\}_{+} \rightarrow\left(X \wedge \triangle[1]_{+}\right) \cup_{X \wedge\{1\}_{+}} Y$ est une cofibration de $\widehat{\mathcal{S}}_{\mathrm{pt}}$.

- Le morphisme $\left(X \wedge \triangle[1]_{+}\right) \cup_{X \wedge\{1\}_{+}} Y \rightarrow Y$ est une équivalence faible de $\widehat{\mathcal{S}}_{\mathrm{pt}}$.

Soient maintenant $X, Y, Z$ des espaces profinis pointés et $X \rightarrow Y$ et $X \rightarrow Z$ des morphismes. On définit la somme amalgamée homotopique du diagramme $Z \leftarrow X \rightarrow Y$ comme la somme amalgamée dans $\widehat{\mathcal{S}}_{\mathrm{pt}}$ du diagramme

$$
\left(X \wedge \triangle[1]_{+}\right) \cup_{X \wedge\{1\}_{+}} Z \leftarrow X \wedge\{0\}_{+} \rightarrow\left(X \wedge \triangle[1]_{+}\right) \cup_{X \wedge\{1\}_{+}} Y .
$$

Le foncteur qui associe au diagramme $Z \leftarrow X \rightarrow Y$ sa somme amalgamée homotopique s'interprète comme le dérivé gauche du foncteur qui associe à ce même diagramme sa colimite dans $\widehat{\mathcal{S}}_{\mathrm{pt}}(\mathrm{cf}[\mathrm{DwS}, 10.7])$.

Supposons enfin que $X \rightarrow Y$ est injective degré par degré (i.e. est une cofibration) et notons $\Sigma$ la somme amalgamée dans $\widehat{\mathcal{S}}_{\text {pt }}$ du diagramme $Z \leftarrow X \rightarrow Y$. Comme dans le cas classique le diagramme obtenu au niveau des complexes de cochaînes continues

$$
\begin{array}{ccc}
\mathrm{C}^{*} \Sigma & \rightarrow & \mathrm{C}^{*} Z \\
\downarrow & & \downarrow \\
\mathrm{C}^{*} Y & \rightarrow & \mathrm{C}^{*} X
\end{array}
$$

est cocartésien de sorte qu'on obtient la suite exacte de Mayer Vietoris en cohomologie modulo $p$ continue.

En utilisant la fonctorialité de cette suite exacte longue et la proposition qui précède on obtient que le morphisme canonique de la somme amalgamée homotopique du diagramme $Z \leftarrow X \rightarrow Y$ dans $\Sigma$ est une équivalence faible.

Cas particulier Soit $X$ un espace profini pointé et prenons pour $Y$ et $Z$ l'espace profini pointé égal au point. Le morphisme $\left(X \wedge \triangle[1]_{+}\right) /\left(X \wedge\{1\}_{+}\right) \rightarrow$ pt induit une équivalence faible de la somme amalgamée homotopique du diagramme pt $\leftarrow X \rightarrow$ pt dans la suspension $\Sigma X$ de $X$. La suite exacte de Mayer Vietoris devient l'isomorphisme en cohomologie modulo $p$ continue réduite

$$
\overline{\mathrm{H}}^{*} \Sigma X \cong \Sigma \overline{\mathrm{H}}^{*} X .
$$




\subsection{Produits fibrés homotopiques}

Dualement notons $X^{\triangle[1]}$ l'espace profini hom $(\triangle[1], X)$. Les morphismes $X \rightarrow$ $X^{\triangle[1]}$ et $X^{\triangle[1]} \rightarrow X \times X$ induits par $\mathrm{d}^{0}, \mathrm{~d}^{1}: \triangle[0] \rightarrow \triangle[1]$ et $\mathrm{s}^{0}: \triangle[1] \rightarrow \triangle[0]$ font de $X^{\triangle[1]}$ un espace de chemins pour $X$ dans $\widehat{\mathcal{S}}$, fonctoriel en $X$. (On peut se convaincre de ce que le morphisme $X \rightarrow X^{\triangle[1]}$ est une équivalence faible en lisant la démonstration de la proposition qui suit.) Tout morphisme $X \rightarrow Y$ entre espaces profinis s'écrit comme la composée des morphismes $X \rightarrow$ $X \times_{Y\{0\}} Y^{\triangle[1]}$ et $X \times_{Y^{\{0\}}} Y^{\triangle[1]} \rightarrow Y^{\{1\}}$.

La proposition suivante est classique pour une catégorie de modèles fermée simpliciale et cruciale pour notre propos :

Proposition 3.2 Soit $X \rightarrow Y$ un morphisme entre espaces profinis ; alors :

- Le morphisme $X \rightarrow X \times_{Y\{0\}} Y^{\triangle[1]}$ est une équivalence faible de $\widehat{\mathcal{S}}$.

- Le morphisme $X \times_{Y\{0\}} Y^{\triangle[1]} \rightarrow Y^{\{1\}}$ est une fibration de $\widehat{\mathcal{S}}$ si $Y$ est fibrant.

Démonstration La démonstration du premier point est analogue à celle de la proposition 5 de [Q, II, $\S 2]$ : Il existe une homotopie simpliciale de la composée $\mathrm{d}^{1} \mathrm{~s}^{0}: \triangle[1] \rightarrow \triangle[1]$ à l'identité de $\triangle[1]$ relativement à $\mathrm{d}^{1}$, c'est à dire un morphisme $\triangle[1] \times \triangle[1] \rightarrow \triangle[1]$ dont la restriction à $\triangle[1] \times\{0\}$ cö̈ncide avec le composé $\triangle[1] \rightarrow\{0\} \rightarrow \triangle[1]$, dont la restriction à $\triangle[1] \times\{1\}$ coïncide avec $\operatorname{Id}_{\triangle[1]}$ et dont la restriction à $\{0\} \times \triangle[1]$ se factorise par la projection $\{0\} \times \triangle[1] \rightarrow\{0\} \times\{0\}$. On en déduit que le morphisme $X \times_{Y^{\{0\}}} Y^{\{0\}} \rightarrow$ $X \times_{Y^{\{0\}}} Y^{\triangle[1]}$ est une équivalence d'homotopie. Or le fait que $X \times \triangle[1]$ soit un objet cylindre pour $X$ implique qu'une équivalence d'homotopie est une équivalence faible.

Pour le deuxième point supposons $Y$ fibrant ; alors le morphisme $Y^{\triangle[1]} \rightarrow$ $Y^{\{0,1\}}$ est une fibration dans $\widehat{\mathcal{S}}$. On en déduit que le morphisme $X \times_{Y^{\{0\}}}$ $Y^{\triangle[1]} \rightarrow X \times_{Y^{\{0\}}} Y^{\{0,1\}} \simeq X \times Y^{\{1\}}$ est une fibration. Maintenant si $X$ est fibrant alors la projection $X \times Y \rightarrow Y$ est une fibration.

On définit la fibre homotopique d'un diagramme $X \rightarrow Y \leftarrow Z$ d'espaces profinis comme le produit fibré dans $\widehat{\mathcal{S}}$ du diagramme

$$
\mathrm{R} X \times_{\mathrm{R} Y\{0\}}(\mathrm{R} Y)^{\triangle[1]} \rightarrow \mathrm{R} Y^{\{1\}} \leftarrow \mathrm{R} Z \times_{\mathrm{R} Y\{0\}}(\mathrm{R} Y)^{\triangle[1]} .
$$

(où $\mathrm{R} X, \mathrm{R} Y$ et $\mathrm{R} Z$ désignent les résolutions fibrantes de $X, Y$ et $Z$ ). Il s'interprète comme le dérivé droit en $X \rightarrow Y \leftarrow Z$ du produit fibré dans $\widehat{\mathcal{S}}$ [DwS, 10.12]. 
Proposition 3.3 Soit $X \rightarrow Y \leftarrow Z$ un diagramme entre espaces profinis. Supposons que $X, Y$ et $Z$ sont fibrants et que le morphisme $Z \rightarrow Y$ est une fibration, alors le morphisme de $X \times_{Y} Z$ dans le produit fibré homotopique de $X \rightarrow Y \leftarrow Z$ est une équivalence d'homotopie.

La proposition est conséquence de la proposition 3.2 et du lemme suivant [GJ, II, lemma 8.10].

Lemme 3.4 Soient $X \rightarrow Y \leftarrow Z$ et $X^{\prime} \rightarrow Y^{\prime} \leftarrow Z^{\prime}$ deux diagrammes entre espaces profinis fibrants et $X \rightarrow X^{\prime}, Y \rightarrow Y^{\prime}, Z \rightarrow Z^{\prime}$ des équivalences faibles commutant avec les morphismes des diagrammes. On suppose que les morphismes $Z \rightarrow Y$ et $X^{\prime} \rightarrow Y^{\prime}$ sont des fibrations de $\widehat{\mathcal{S}}$. Alors le morphisme $X \times_{Y} Z \rightarrow X^{\prime} \times_{Y^{\prime}} Z^{\prime}$ est une équivalence faible.

Cas particulier Soit $Y$ un espace profini pointé fibrant et prenons pour $X$ et $Z$ l'espace profini pointé égal au point. Les propositions 3.2 et 3.3 montrent que le morphisme pt $\rightarrow$ pt $\times_{Y^{\{0\}}} Y^{\triangle[1]}$ induit une équivalence faible de l'espace de lacets $\Omega Y$ dans le produit fibré homotopique du diagramme pt $\rightarrow Y \leftarrow$ pt.

Notre stratégie pour étudier la cohomologie modulo $p$ continue d'un produit fibré homotopique est de comparer celui-ci avec la limite d'un diagramme filtrant de produits fibrés d'espaces $p$-finis auquel on associera un diagramme de suites spectrales d'Eilenberg-Moore.

Précisons : On associe d'abord au diagramme $X \rightarrow Y \leftarrow Z$ le diagramme des pro- $p$-complétés $\widehat{X}(-) \rightarrow \widehat{Y}(-) \leftarrow \widehat{Z}(-)$. On modifie ensuite les catégories index de $\widehat{X}(-), \widehat{Y}(-)$ et $\widehat{Z}(-)$ de sorte que les morphismes $\widehat{X}(-) \rightarrow \widehat{Y}(-)$ et $\widehat{Z}(-) \rightarrow \widehat{Y}(-)$ soient des transformations naturelles entre diagrammes filtrants d'espaces (cf [AM, appendice, §3]). Nous en rappelons l'argument :

Soient $X(-)$ un pro-objet de catégorie index $\mathcal{I}, \mathcal{J}$ une autre catégorie petite et filtrante et $\phi: \mathcal{J} \rightarrow \mathcal{I}$ un foncteur. Les identités de $X(\phi(j)), j$ décrivant $\mathcal{J}$, induisent un morphisme de pro-objets $X(-) \rightarrow X \circ \phi(-)$.

Lemme 3.5 Soit $X(-) \rightarrow Y(-)$ un morphisme entre pro-objets. Notons $\mathcal{I}_{X}$ et $\mathcal{I}_{Y}$ leurs catégories index respectives. Il existe une petite catégorie filtrante $\mathcal{J}$, des foncteurs $\phi: \mathcal{J} \rightarrow \mathcal{I}_{X}$ et $\psi: \mathcal{J} \rightarrow \mathcal{I}_{Y}$ et une transformation naturelle $X \circ \phi \rightarrow Y \circ \psi$ tels que les morphismes $X(-) \rightarrow X \circ \phi(-)$ et $Y(-) \rightarrow Y \circ \psi(-)$ soient des pro-isomorphismes et tels que le diagramme de pro-objets

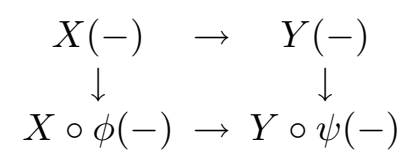

commute. 
Démonstration Soit $\mathcal{J}$ la catégorie définie comme suit :

- ses objets sont les triplets $(i, j, X(i) \rightarrow Y(j)),(i, j)$ décrivant $\mathcal{I}_{X} \times \mathcal{I}_{Y}$, tels que le morphisme $X(i) \rightarrow Y(j)$ représente le morphisme $X(-) \rightarrow$ $Y(j)$,

- un morphisme d'un objet $(i, j, X(i) \rightarrow Y(j))$ dans un objet $\left(i^{\prime}, j^{\prime}, X\left(i^{\prime}\right) \rightarrow\right.$ $\left.Y\left(j^{\prime}\right)\right)$ est un couple de morphismes $\left(i \rightarrow i^{\prime}, j \rightarrow j^{\prime}\right)$ tels que le diagramme

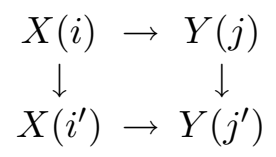

commute.

On note $\phi$ et $\psi$ les foncteurs évidents $\mathcal{J} \rightarrow \mathcal{I}_{X}$ et $\mathcal{J} \rightarrow \mathcal{I}_{Y}$. Alors $\mathcal{J}$ est une catégorie petite et filtrante, les images de $\mathcal{J}$ dans $\mathcal{I}_{X}$ et $\mathcal{I}_{Y}$ sont cofinales de sorte que les morphismes $X(-) \rightarrow X \circ \phi(-)$ et $Y(-) \rightarrow Y \circ \psi(-)$ sont des pro-isomorphismes ( $\mathrm{cf}[\mathrm{AM}$, Appendice, $\S 3]$ ) et on dispose d'une transformation naturelle $X \circ \phi \rightarrow Y \circ \psi$ satisfaisant aux conditions de l'énoncé.

Observons que, $\phi$ étant fixé, le $\mathcal{J}$-diagramme $X \circ \phi$ est fonctoriel en le $\mathcal{I}_{X}$ diagramme $X$. En itérant le procédé on voit que si $X(-) \rightarrow Y(-) \leftarrow Z(-)$ est un diagramme entre pro-objets, il existe une catégorie filtrante $\mathcal{J}$, des foncteurs $\phi: \mathcal{J} \rightarrow \mathcal{I}_{X}, \psi: \mathcal{J} \rightarrow \mathcal{I}_{Y}$ et $\theta: \mathcal{J} \rightarrow \mathcal{I}_{Z}$ induisant des pro-isomorphismes $X(-) \rightarrow X \circ \phi(-)$, etc. et il existe des transformations naturelles $X \circ \phi \rightarrow Y \circ \psi$ et $Z \circ \theta \rightarrow Y \circ \psi$ tels que le diagramme

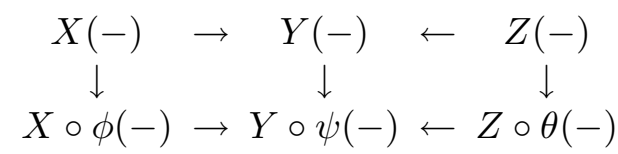

soit commutatif.

Revenons au produit fibré homotopique d'un diagramme $X \rightarrow Y \leftarrow Z$ d'espaces profinis. Ce qui précède permet de conclure que le diagramme $\mathrm{R} X \rightarrow \mathrm{R} Y \leftarrow$ $\mathrm{R} Z$ est limite filtrante de diagrammes $\widehat{X}(\phi(j)) \rightarrow \widehat{Y}(\psi(j)) \leftarrow \widehat{Z}(\theta(j))$ entre espaces $p$-finis. Le produit fibré du diagramme

$$
\mathrm{R} X \times_{\mathrm{R} Y} \mathrm{R} Y^{\triangle[1]} \rightarrow \mathrm{R} Y \leftarrow \mathrm{R} Z \times_{\mathrm{R} Y} \mathrm{R} Y^{\triangle[1]}
$$

est alors la limite filtrante des produits fibrés des diagrammes entre espaces $p$-finis

$$
\widehat{X}(\phi(j)) \times_{\widehat{Y}(\psi(j))} \widehat{Y}(\psi(j))^{\triangle[1]} \rightarrow \widehat{Y}(\psi(j)) \leftarrow \widehat{Z}(\theta(j)) \times_{\widehat{Y}(\psi(j))} \widehat{Y}(\psi(j))^{\triangle[1]} .
$$

Nous somme prêts à mettre en place la suite spectrale d'Eilenberg-Moore. 


\section{Suite spectrale d'Eilenberg-Moore et cohomologie continue des espaces de lacets}

\subsection{Construction}

Nous suivons l'approche géométrique de Rector [R], voir aussi [Bou] pour une comparaison avec la construction classique.

Soit $X \rightarrow Y \leftarrow Z$ un diagramme entre espaces profinis ; on lui associe le diagramme cosimplicial d'espaces profinis donné par la construction cobar géométrique, qu'on note $\mathrm{B}^{\bullet}(X \rightarrow Y \leftarrow Z)$ ou plus simplement $\mathrm{B}^{\bullet}$ lorsqu'il n'y a pas d'ambiguité : $\mathrm{B}^{n}=X \times Y^{n} \times Z$ pour $n \geq 0$, et la coaugmentation $X \times_{Y} Z \rightarrow \mathrm{B}^{0}(\operatorname{cf}[\mathrm{R}])$.

Rappelons qu'on définit le complexe normalisé $\mathrm{N}_{*} A$ d'un objet simplicial $A$. d'une catégorie abélienne comme le complexe ayant pour objet en degré $n$ l'intersection des noyaux des faces $\mathrm{d}_{i}, 1 \leq i \leq n$, et pour différentielle le morphisme induit par $\mathrm{d}_{0}$. Ce complexe est isomorphe au complexe ayant pour objet en degré $n$ le quotient de $A_{n}$ par l'image des dégénérescences et pour différentielle le morphisme induit par la somme alternée des faces. Le $n$-ième objet d'homologie $\mathrm{H}_{n} \mathrm{~N} A$ du complexe $\mathrm{N}_{*} A$ s'identifie au $n$-ième objet d'homotopie $\pi_{n} A$ de l'objet simplicial $A_{\bullet}$ et est isomorphe au $n$-ième objet d'homologie du complexe $\left(A_{n}, \sum(-1)^{i} \mathrm{~d}_{i}\right)$. (On a une équivalence d'homotopie canonique entre les complexes $\mathrm{N}_{*} A$ et $A_{*}$.) Voir par exemple [May, §22].

On définit les espaces profinis pointés $\mathrm{N}^{0} \mathrm{~B}=\mathrm{B}_{+}^{0}$ et $\mathrm{N}^{n} \mathrm{~B}=\mathrm{B}^{n} /\left(\operatorname{Im}\left(\mathrm{d}^{1}\right) \cup \ldots \cup\right.$ $\left.\operatorname{Im}\left(\mathrm{d}^{n-1}\right)\right)$ pour $n \geq 1$. Le morphisme $\mathrm{d}^{0}: \mathrm{B}^{n} \rightarrow \mathrm{B}^{n+1}$ induit un morphisme $\mathrm{N}^{n} \mathrm{~B} \rightarrow \mathrm{N}^{n+1} \mathrm{~B}$ telle que le composé $\mathrm{N}^{n} \mathrm{~B} \rightarrow \mathrm{N}^{n+1} \mathrm{~B} \rightarrow \mathrm{N}^{n+2} \mathrm{~B}$ est le morphisme constant. Le complexe induit en cohomologie continue réduite par la suite $\mathrm{N}^{0} \mathrm{~B} \rightarrow \mathrm{N}^{1} \mathrm{~B} \rightarrow \cdots$ s'identifie au normalisé $\mathrm{N}_{*} \mathrm{H}^{*} \mathrm{~B}$ du groupe abélien gradué simplicial $\mathrm{H}^{*} \mathrm{~B}^{\bullet}$.

On construit ensuite inductivement une suite d'espaces profinis pointés $X^{n}$ comme suit : On pose $X^{-1}=\left(X \times_{Y} Z\right)_{+}$. La composée $X^{-1} \rightarrow \mathrm{N}^{0} \mathrm{~B} \rightarrow \mathrm{N}^{1} \mathrm{~B}$ est le morphisme constant. Supposons construit $X^{n-1}$ avec un morphisme $X^{n-1} \rightarrow \mathrm{N}^{n} \mathrm{~B}$ telle que la composée avec $\mathrm{N}^{n} \mathrm{~B} \rightarrow \mathrm{N}^{n+1} \mathrm{~B}$ soit constante. Notons $N^{\prime n}$ l'espace profini pointé $\left(X^{n-1} \wedge \triangle[1]_{+}\right) \cup_{X^{n-1}} \wedge\{1\}_{+} \mathrm{N}^{n} \mathrm{~B}$. Le morphisme $X^{n-1} \rightarrow \mathrm{N}^{n} \mathrm{~B}$ s'écrit comme la composé de la cofibration $X^{n-1} \rightarrow N^{\prime n}$ avec l'équivalence faible $N^{\prime n} \rightarrow \mathrm{N}^{n} \mathrm{~B}$. On définit $X^{n}$ comme le quotient de $N^{\prime n}$ par $X^{n-1}$. La composée $N^{\prime n} \rightarrow \mathrm{N}^{n} \mathrm{~B} \rightarrow \mathrm{N}^{n+1} \mathrm{~B}$ se factorise par $N^{\prime n} \rightarrow X^{n}$ et $X^{n} \rightarrow \mathrm{N}^{n+1} \mathrm{~B}$ vérifie l'hypothèse de récurrence. 
On obtient ainsi une suite de cofibrations $X^{n-1} \rightarrow N^{\prime n} \rightarrow X^{n}$ entre espaces profinis pointés et d'équivalences faibles $N^{\prime n} \rightarrow \mathrm{N}^{n} \mathrm{~B}$.

Posons $A^{-s, *}=\overline{\mathrm{H}}^{*+s+1} X^{s}$ pour $-s \leq 1, A^{-s, *}=A^{1, *}=\overline{\mathrm{H}}^{*}\left(X \times_{Y} Z\right)_{+}$pour $-s>1$ et $E_{1}^{-s, *}=\overline{\mathrm{H}}^{*+s} \mathrm{~N}^{s} B$.

On a un triangle exact

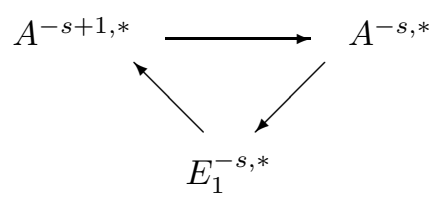

où $A^{-s, *} \rightarrow E_{1}^{-s, *}$ est un morphisme de bidegré $(0,1)$, donc une suite spectrale $\left(E_{r}, \mathrm{~d}_{r}: E_{r}^{-s, t} \rightarrow E_{r}^{-s+r, t+1}\right)$ vérifiant : (Cf les lemmes 5.6, 5.9 et le théorème 6.1 de $[\mathrm{Boa}]$.

- Le terme $E_{1}^{s}$ est nul pour tout $s>0$ donc la suite $\left(E_{r}^{-s}\right)_{r}$ est une suite d'épimorphismes pour $r>s$. On pose $E_{\infty}^{-s}=\operatorname{colim}_{r} E_{r}^{-s}$.

- Le groupe abélien gradué $A^{1} \cong \mathrm{H}^{*} X \times_{Y} Z$ a une filtration naturelle $0 \subset \mathrm{F}_{0} A^{1} \subset \mathrm{F}_{-1} A^{1} \subset \cdots \subset A^{1}$ définie par $\mathrm{F}_{-s}=\operatorname{Ker}\left(A^{1} \rightarrow A^{-s}\right)$. On a un épimorphisme $E_{\infty}^{-s} \rightarrow \mathrm{F}_{-s} A^{1} / \mathrm{F}_{-s+1} A^{1}$ et en particulier un morphisme de coin $E_{r}^{0} \rightarrow \mathrm{F}_{0} A^{1} \subset A^{1}$.

- Les conditions suivantes sont équivalentes :

(1) La filtration de $A^{1}$ est exhaustive et le morphisme $E_{\infty}^{-s} \rightarrow$ $\mathrm{F}_{-s} A^{1} / \mathrm{F}_{-s+1} A^{1}$ est un isomorphisme pour tout $s$.

(2) Le groupe abélien gradué $\operatorname{colim}_{s} A^{-s}$ est nul.

On réindexe la suite spectrale en posant $\mathrm{E}_{r}^{-s, t}=E_{r}^{-s, t+s}$ de sorte que le terme $\mathrm{E}_{1}$ est donné par

$$
\mathrm{E}_{1}^{-s, t}=\overline{\mathrm{H}}^{t} \mathrm{~N}^{s} \mathrm{~B} \cong \mathrm{N}_{s} \mathrm{H}^{t} \mathrm{~B}
$$

et la différentielle $\mathrm{d}_{r}$ est de bidegré $(r, 1-r)$.

On appelle cette suite spectrale la suite spectrale d'Eilenberg-Moore en cohomologie modulo $p$ continue associée au diagramme $X \rightarrow Y \leftarrow Z$.

\subsection{Convergence}

Le théorème 3.1 de [Sh], qui s'appuie sur le théorème de convergence forte de Dwyer $[\mathrm{Dw}]$, entraîne la suivante :

Algebraic 83 Geometric Topology, Volume 3 (2003) 
Proposition 4.1 Soit $X \rightarrow Y \leftarrow Z$ un diagramme entre espaces $p$-finis tel que $Z \rightarrow Y$ est une fibration, alors la suite spectrale d'Eilenberg-Moore associée converge fortement vers la cohomologie modulo $p$ du produit fibré $X \times_{Y} Z$.

Soit maintenant $X \rightarrow Y \leftarrow Z$ un diagramme entre espaces profinis quelconques. Le morphisme de ce diagramme dans sa "résolution fibrante" $\mathrm{R} X \times_{\mathrm{R} Y} \mathrm{R} Y \triangle[1] \rightarrow$ $\mathrm{R} Y \leftarrow \mathrm{R} Z \times_{\mathrm{R} Y} \mathrm{R} Y^{\triangle[1]}$ induit un isomorphisme au niveau des termes $\mathrm{E}_{2}$ des suites spectrales d'Eilenberg-Moore. La proposition qui précède, la discussion sur les produits fibrés et l'exactitude des colimites filtrantes entraînent :

Proposition 4.2 Soit $X \rightarrow Y \leftarrow Z$ un diagramme entre espaces profinis. La suite spectrale d'Eilenberg-Moore associée converge vers la cohomologie modulo $p$ continue de son produit fibré homotopique.

Cas particulier Soit $X$ un espace profini pointé fibrant. La suite spectrale d'Eilenberg-Moore associée au diagramme pt $\rightarrow X \leftarrow$ pt converge vers la cohomologie modulo $p$ continue de l'espace profini de lacets $\Omega X$.

\subsection{Structure de la suite spectrale d'Eilenberg-Moore}

Rappelons que pour $X$ et $Y$ deux espaces profinis on dispose d'un isomorphisme canonique $\mathrm{H}^{*} X \otimes \mathrm{H}^{*} Y \rightarrow \mathrm{H}^{*} X \times Y$.

L'objet gradué simplicial $\mathrm{H}^{*} \mathrm{~B}^{\bullet}$ s'identifie donc au produit tensoriel au dessus de $\mathrm{H}^{*} Y$ de $\mathrm{H}^{*} X$ avec la résolution simplicial canonique de $\mathrm{H}^{*} Z$ comme $\mathrm{H}^{*} Y$ module. Notons $\overline{\mathrm{H}}^{*} Y$ le conoyau de l'unité $\mathbb{F}_{p} \rightarrow \mathrm{H}^{*} Y$. Le complexe normalisé $\mathrm{N}_{*} \mathrm{H}^{*} \mathrm{~B}$ s'identifie au produit tensoriel au dessus de $\mathrm{H}^{*} Y$ de $\mathrm{H}^{*} X$ avec la résolution bar réduite de $\mathrm{H}^{*} Z$ comme $\mathrm{H}^{*} Y$-module (cf [Mac, Chap. X, §2]). Autrement dit on a un isomorphisme canonique

$$
\mathrm{E}_{1}^{-s, *} \cong \mathrm{H}^{*} X \otimes\left(\overline{\mathrm{H}}^{*}\right)^{\otimes s} \otimes \mathrm{H}^{*} Z .
$$

Le terme $\mathrm{E}_{2}^{-s, *}$ s'identifie à l'objet $\operatorname{Tor}_{s}^{\mathrm{H}^{*} Y}\left(\mathrm{H}^{*} X, \mathrm{H}^{*} Z\right)$ et on a un morphisme de coin

$$
\mathrm{E}_{2}^{0, *} \cong \mathrm{H}^{*} X \otimes_{\mathrm{H}^{*} Y} \mathrm{H}^{*} Z \rightarrow \mathrm{E}_{\infty}^{0, *} \rightarrow \mathrm{F}_{0} \mathrm{H}^{*} X \times_{Y} Z \subset \mathrm{H}^{*} X \times_{Y} Z .
$$

Cas particulier Supposons $X=Z=$ pt et $Y$ fibrant. Le morphisme $X \rightarrow Y$ munit $Y$ d'un point base et on a un isomorphisme canonique $\overline{\mathrm{H}}^{*} Y \cong$ $\operatorname{Ker}\left(\mathrm{H}^{*} Y \rightarrow \mathrm{H}^{*} \mathrm{pt}\right)$. Le terme $\mathrm{E}_{1}^{-s, *}$ s'identifie à $\left(\overline{\mathrm{H}}^{*} Y\right)^{\otimes s}$. La différentielle $\mathrm{d}_{1}: \mathrm{E}_{1}^{-1, *} \rightarrow \mathrm{E}_{1}^{0, *}=\mathbb{F}_{p}$ est nulle. La différentielle $\mathrm{d}_{1}: \mathrm{E}_{1}^{-2, *} \rightarrow \mathrm{E}_{1}^{-1, *}$ est 
donnée par le cup produit restreint à $\overline{\mathrm{H}}^{*} Y$. La filtration de la cohomologie continue de $\Omega Y$ induit une filtration de la cohomologie continue réduite de $\Omega Y$ et on dispose de morphismes de coin

$$
\mathbb{F}_{p}=\mathrm{E}_{1}^{0, *}=\mathrm{E}_{\infty}^{0, *} \cong \mathrm{F}_{0} \mathrm{H}^{*} \Omega Y=\mathbb{F}_{p}
$$

et

$$
\overline{\mathrm{H}}^{*} Y \cong \mathrm{E}_{1}^{-1, *} \rightarrow \mathrm{E}_{\infty}^{-1, *} \cong \Sigma \mathrm{F}_{-1} \overline{\mathrm{H}}^{*} \Omega Y
$$

Venons en à la structure de module sur l'algèbre de Steenrod.

Le triangle exact

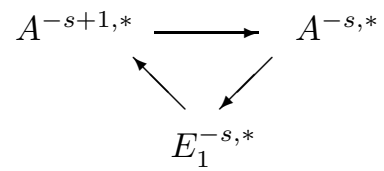

dont est issue la suite spectrale est un triangle exact de modules instables sur l'algèbre de Steenrod. Les groupes abéliens gradués $\mathrm{E}_{r}^{-s, *}$ sont donc des modules instables pour tous $r, s$ de façon compatible avec l'aboutissement et la différentielle $\mathrm{d}_{r}: \mathrm{E}_{r}^{-s, *} \rightarrow \Sigma^{r-1} \mathrm{E}_{r}^{-s+r, *}$ est un morphisme de modules instables.

Donnons nous maintenant deux diagrammes $X \rightarrow Y \leftarrow Z$ et $X^{\prime} \rightarrow Y^{\prime} \leftarrow Z^{\prime}$ entre espaces profinis et formons leur produit $X \times X^{\prime} \rightarrow Y \times Y^{\prime} \leftarrow Z \times Z^{\prime}$.

La suite spectrale d'Eilenberg-Moore associée au diagramme $X \times X^{\prime} \rightarrow Y \times$ $Y^{\prime} \leftarrow Z \times Z^{\prime}$ s'identifie au produit tensoriel des suites spectrales associées à $X \rightarrow Y \leftarrow Z$ et $X^{\prime} \rightarrow Y^{\prime} \leftarrow Z^{\prime}$. En particulier pour tout diagramme $X \rightarrow Y \leftarrow Z$, les diagonales $X \rightarrow X \times X, Y \rightarrow Y \times Y$, etc. font de la suite spectrale associée au diagramme une suite spectrale de $\mathrm{F}_{p}$-algèbres bigraduées compatible avec la structure multiplicative de l'aboutissement.

Remarque Chaque espace profini formant le diagramme $X \rightarrow Y \leftarrow Z$ est la limite filtrante de ses quotients finis simpliciaux. En utilisant le lemme 3.5 on obtient que le diagramme lui même est la limite filtrante de diagrammes $X(i) \rightarrow Y(i) \leftarrow Z(i)$ entre ensembles finis simpliciaux. La suite spectrale d'Eilenberg-Moore en cohomologie modulo $p$ associée à $X \rightarrow Y \leftarrow Z$ s'identifie alors à la colimite (filtrante) des suites spectrales d'Eilenberg Moore classiques associées aux diagrammes $X(i) \rightarrow Y(i) \leftarrow Z(i)$ de façon compatible avec sa structure. 


\section{Rappels sur la cohomologie modulo $p$ des espaces fonctionnels de source le classifiant du groupe cy- clique d'ordre $p$}

On note $\mathrm{B} \mathbb{Z} / p$ le classifiant du groupe cyclique d'ordre $p$. C'est un ensemble fini simplicial représentant le foncteur qui associe à un espace profini l'ensemble de ses 1-cocycles continus à coefficients dans $\mathbb{Z} / p$.

Rappelons que $\mathcal{U}$ désigne la catégorie des modules instables sur l'algèbre de Steenrod modulo $p$ et notons $\mathrm{H}$ la cohomologie modulo $p$ de $\mathrm{B} \mathbb{Z} / p$. Le foncteur $\mathcal{U} \rightarrow \mathcal{U}, M \mapsto \mathrm{H} \otimes M$ admet un adjoint à gauche noté $\mathrm{T}$ (voir [La]). Pour tout espace profini $X$, l'évaluation $\mathrm{B} \mathbb{Z} / p \times \operatorname{hom}(\mathrm{B} \mathbb{Z} / p, X) \rightarrow X$ induit un morphisme naturel $\mathrm{TH}^{*} X \rightarrow \mathrm{H}^{*} \operatorname{hom}(\mathrm{B} \mathbb{Z} / p, X)$.

Théorème 5.1 ([La], $[\mathrm{DrS}])$ Pour tout espace $p$-fini $X$ le morphisme $\mathrm{TH}^{*} X \rightarrow \mathrm{H}^{*} \operatorname{hom}(\mathrm{BZ} / p, X)$ est un isomorphisme.

Morel en déduit le théorème suivant :

Théorème 5.2 [Mo] Soit $X$ un espace profini fibrant; alors le morphisme $\mathrm{TH}^{*} X \rightarrow \mathrm{H}^{*} \operatorname{hom}(\mathrm{BZ} / p, X)$ est un isomorphisme.

Indiquons comment le dernier théorème se déduit du précédent : on observe d'abord que lorsque $X$ est fibrant dans $\widehat{\mathcal{S}}$ le morphisme de $X$ dans sa résolution fibrante $\mathrm{R} X=\lim \widehat{X}(-)$ est une équivalence d'homotopie simpliciale. On en déduit que pour $X$ fibrant le morphisme hom(BZ $/ p, X) \rightarrow$ $\operatorname{hom}(\mathrm{B} \mathbb{Z} / p, \mathrm{R} X)$ est une équivalence d'homotopie donc induit un isomorphisme en cohomologie modulo $p$ continue. On observe ensuite que le morphisme $\mathrm{TH}^{*} \mathrm{R} X \rightarrow \mathrm{H}^{*} \operatorname{hom}(\mathrm{B} \mathbb{Z} / p, \mathrm{R} X)$ est la colimite des morphismes $\mathrm{TH}^{*} \widehat{X}(i) \rightarrow$ $\mathrm{H}^{*} \operatorname{hom}(\mathrm{BZ} / p, \widehat{X}(i))$ où les $\widehat{X}(i)$ sont des espaces $p$-finis.

Soit maintenant $\overline{\mathrm{H}}$ la cohomologie modulo $p$ réduite de $\mathrm{B} \mathbb{Z} / p$. Le foncteur $\mathcal{U} \rightarrow \mathcal{U}, M \mapsto \overline{\mathrm{H}} \otimes M$ admet également un adjoint à gauche, le foncteur de Lannes réduit noté $\overline{\mathrm{T}}$. La décomposition $\mathrm{H}=\mathbb{F}_{p} \oplus \overline{\mathrm{H}}$ induit pour toute module instable $M$ un scindement canonique :

$$
\mathrm{T} M=M \oplus \overline{\mathrm{T}} M .
$$

On peut comme précédemment donner une interprétation géométrique de $\overline{\mathrm{T}}$ :

Soit $X$ un espace profini, $\mathrm{R} X$ le remplacement fibrant de $X$ et $Y$ la cofibre du morphisme $\mathrm{R} X \rightarrow \operatorname{hom}(\mathrm{B} \mathbb{Z} / p, \mathrm{R} X)$ induit par le morphisme $\mathrm{B} \mathbb{Z} / p \rightarrow$ pt. 
L'inclusion du point dans $\mathrm{B} \mathbb{Z} / p$ fait de $\mathrm{R} X$ un rétracte de $\mathbf{h o m}(\mathrm{B} \mathbb{Z} / p, \mathrm{R} X)$ de sorte qu'on a une suite exacte courte

$$
0 \rightarrow \overline{\mathrm{H}}^{*} Y \rightarrow \mathrm{H}^{*} \operatorname{hom}(\mathrm{BZ} / p, \mathrm{R} X) \rightarrow \mathrm{H}^{*} X \rightarrow 0 .
$$

L'isomorphisme $\mathrm{TH}^{*} X \rightarrow \mathrm{H}^{*} \operatorname{hom}(\mathrm{BZ} / p, \mathrm{R} X)$ induit alors un isomorphisme naturel

$$
\overline{\mathrm{T}} \mathrm{H}^{*} X \rightarrow \overline{\mathrm{H}}^{*} Y .
$$

Corollaire 5.3 Soit $M$ un module instable. Si $M$ est la cohomologie continue réduite d'un espace profini $X$, il est de même pour $\overline{\mathrm{T}} M$.

\section{$6 \quad$ Filtrations de la catégorie $\mathcal{U}$ (voir $[\mathrm{Sc1}]$ et $[\mathrm{Ku}]$ )}

Dans toute cette section, on se spécialise au cas $p=2$.

Rappelons qu'une sous-catégorie abélienne $\mathcal{D}$ d'une catégorie abélienne $\mathcal{C}$ est dite de Serre, si pour toute suite exacte courte $A \longrightarrow B \longrightarrow C$ telle que $A$ et $C$ sont dans $\mathcal{D}$, alors $B$ est également dans $\mathcal{D}$.

\subsection{La filtration nilpotente}

Soit $M$ un module instable et $m$ un entier naturel. Pour tout élément $x$ de $M$, on note $|x|$ le degré de $x$. On définit des opérateurs

$$
\mathrm{Sq}_{m}: M \longrightarrow M, x \longmapsto \mathrm{Sq}^{|x|-m} x .
$$

On dit qu'un élément $x \in M$ est $s$-nilpotent si :

$$
\forall 0 \leq t<s, \exists c \in \mathbb{N},\left(\mathrm{Sq}_{t}\right)^{c} x=0 .
$$

Dans le cas où $x$ est $s$-nilpotent, mais n'est pas $(s+1)$-nilpotent, on dit que $x$ est exactement $s$-nilpotent.

Un élément exactement 0 -nilpotent est dit réduit. Un module instable est dit réduit si tous ses éléments sont réduits. En termes plus simples, un module instable $M$ est réduit si et seulement si l'application

$$
\mathrm{Sq}_{0}: M \longrightarrow M, x \longmapsto \mathrm{Sq}^{|x|} x
$$

est injective. Dans le cas où $M$ est muni d'une structure d'algèbre compatible, l'élévation au carré cö̈ncide avec $\mathrm{Sq}_{0}$ et $M$ est réduit comme module instable si et seulement si $M$ est réduit comme algèbre, i.e. n'a pas d'éléments nilpotents. 
Un module instable est $s$-nilpotent si tous ses éléments sont au moins $s$ nilpotents. La sous-catégorie pleine de $\mathcal{U}$ dont les objets sont les modules $s$-nilpotents est de Serre et stable par colimites. Cette sous-catégorie, notée $\mathcal{N} i l_{s}$, coïncide avec la plus petite sous-catégorie de Serre stable par colimite qui contient tous les modules qui sont des suspensions $s$-ièmes.

Soit $M$ un module instable. On définit $M_{s}$ comme le plus grand sous-module de $M$ qui est dans $\mathcal{N} i l_{s}$. Les sous-modules $\left\{M_{s}\right\}_{s \in \mathbb{N}}$ forment une filtration décroissante naturelle et séparée de $M$. Le sous-quotient $M_{s} / M_{s+1}$ est la suspension $s$-ième $\Sigma^{s} \mathrm{R}_{s} M$ d'un module réduit $\mathrm{R}_{s} M$.

Soient $M$ et $N$ deux modules instables. On a $(M \otimes N)_{n} \cong \oplus_{i+j=n} M_{i} \otimes N_{j}$. Par conséquent, on a pour tout $n$ la formule $R_{n}(M \otimes N) \cong \oplus_{i+j=n} \mathrm{R}_{i} M \otimes \mathrm{R}_{j} N$ (voir $[\mathrm{Ku}$, Proposition 2.5, (1)]). En particulier on a un isomorphisme naturel $\mathrm{R}_{n} \Sigma M \cong \mathrm{R}_{n-1} M$.

On dispose également d'une caractérisation du degré de nilpotence d'un module instable en terme de foncteur de Lannes: un module instable $M$ est $s$-nilpotent si et seulement si pour tout $n, \mathrm{~T}^{n} M$ est $(s-1)$-connexe ( $c f$ [Sc1, DéfinitionProposition 6.1.1, p. 139]).

\subsection{La filtration de Krull}

La filtration de Krull $\left\{\mathcal{U}_{n}\right\}_{n \in \mathbb{N}}$ de la catégorie $\mathcal{U}$ est définie par : $\mathcal{U}_{n}$ est la sous-catégorie pleine de $\mathcal{U}$ formée des objets annulés par $\overline{\mathrm{T}}^{n}$.

C'est une filtration croissante par des sous-catégories de Serre stables par colimites et suspensions, qui est exhaustive au sens où la plus petite sous-catégorie de $\mathcal{U}$ stable par colimite et contenant tous les $\mathcal{U}_{n}$ pour tout entier $n$ est $\mathcal{U}$ elle-même.

La filtration de Krull induit sur chaque module instable une filtration croissante naturelle et complète par des sous-modules qui sont dans $\mathcal{U}_{n}$. Dans le cas des modules réduits, cette filtration coïncide avec la filtration par le poids, dont la définition suit.

Définition 6.1 (Poids d'un module réduit) (1) Soit $n$ un entier naturel, on définit $\alpha(n)$ comme le nombre de 1 dans l'écriture diadique de $n$.

(2) Un module instable réduit est de poids inférieur ou égal à $n$ s'il est nul dans les degrés $i$ tels que $\alpha(i)>n$. 
Un module réduit est filtré par ses sous-modules de poids $n$ maximaux. Cette filtration est exhaustive. Le poids d'un module instable réduit $M$ est noté $w(M)$. La caractérisation suivante est extraite de [FS].

Proposition 6.2 (Voir [FS]) Un module réduit est dans $\mathcal{U}_{n}$ si et seulement s'il est de poids inférieur ou égal à $n$.

Remarquons enfin que si $M$ est dans $\mathcal{U}_{n}$ et si $N$ est dans $\mathcal{U}_{m}$, alors $M \otimes N$ est dans $\mathcal{U}_{n+m}$.

\section{Démonstration du théorème 1.2}

Dans cette section, on fixe $p=2$.

On démontre dans cette section le théorème 1.2 en reprenant la preuve de L. Schwartz. Ceci démontre la conjecture de non réalisation forte pour $p=2$ en toute généralité puisque la cohomologie modulo 2 d'un espace est aussi la cohomologie modulo 2 continue de son complété profini.

On suppose qu'il existe un espace profini dont la cohomologie modulo 2 continue n'est pas localement finie, et qui est dans cran fini de la filtration de Krull. On va trouver une contradiction. La première étape dans ce sens est la réduction de $N$. Kuhn: on va montrer qu'il existe alors un espace profini dont la cohomologie continue est dans $\mathcal{U}_{1}$ mais n'est pas dans $\mathcal{U}_{0}$ (i.e. n'est pas localement finie).

\subsection{La réduction de N. Kuhn}

Proposition 7.1 (Réduction de Kuhn) S'il existe un entier $n \geq 1$ et un espace profini dont la cohomologie modulo 2 continue est dans $\mathcal{U}_{n}$ mais n'est pas dans $\mathcal{U}_{n-1}$, alors il existe un espace profini dont la cohomologie modulo 2 continue est dans $\mathcal{U}_{1}$ mais n'est pas dans $\mathcal{U}_{0}$.

Démonstration Soit $X$ un espace profini dont la cohomologie continue $M$ est dans $\mathcal{U}_{n}$ mais n'est pas dans $\mathcal{U}_{n-1}$ et supposons $n>1$. Alors $\overline{\mathrm{T}} M$ est dans $\mathcal{U}_{n-1}$ mais pas dans $\mathcal{U}_{n-2}$, et est la cohomologie continue réduite d'un espace profini d'après le corollaire 5.3. On montre ainsi l'énoncé par une récurrence descendante. 


\subsection{Construction des classes $\alpha_{i, d}$ (d'après [Sc2])}

On s'est ramené à considérer un espace profini $X$ dont la cohomologie continue est dans $\mathcal{U}_{1}$ mais n'est pas localement finie. On utilise la structure très particulière de ces modules pour construire des classes de la cohomologie continue d'un espace de lacets itérés de $X$ qui ont des propriétés contradictoires.

Soit $X$ un espace profini dont la cohomologie continue est dans $\mathcal{U}_{1}$ mais n'est pas localement finie. Le module instable $\overline{\mathrm{T}} \mathrm{H}^{*} X$ est non nul par définition. Soit $(d-1)$ sa connexité.

Remarquons que si un espace profini $X$ a sa cohomologie continue dans $\mathcal{U}_{1}$ mais pas dans $\mathcal{U}_{0}$, il en est de même pour toutes ses suspensions. En effet, le foncteur $\overline{\mathrm{T}}$ est exact et commute aux suspensions et aux colimites.

On peut donc supposer que $X$ est une suspension et, par conséquent, on peut supposer que $d \geq 1$ et que tous les cup-produits sont nuls dans la cohomologie continue réduite de $X$.

On définit pour tout entier $n$ le $n$-squelette $\operatorname{Sk}_{n} Z$ d'un espace profini $Z$ comme le sous-espace profini engendré par les simplexes non dégénérés de dimension inférieure ou égale à $n$. Si $Z$ est la limite d'un diagramme filtrant d'ensembles finis simpliciaux $Z(-)$, alors $\operatorname{Sk}_{n} Z$ est la limite des $n$-squelettes des $Z(i)$.

On voit que la cohomologie continue réduite du quotient de $X$ par son $(d-1)$ squelette est un module instable $(d-1)$-connexe qui ne diffère de la cohomologie continue de $X$ que par un module borné, donc localement fini. Par conséquent $\overline{\mathrm{T}} \mathrm{H}^{*} X=\overline{\mathrm{T}} \overline{\mathrm{H}}^{*}\left(X / \mathrm{Sk}_{d-1} X\right)$. Ainsi $X / \mathrm{Sk}_{d-1} X$ est également un espace profini dont la cohomologie continue est dans $\mathcal{U}_{1}$ mais n'est pas localement finie. De plus $X / \mathrm{Sk}_{d-1} X$ est encore une suspension donc tous les cup-produits sont nuls dans sa cohomologie continue réduite.

On peut donc aussi supposer que $\overline{\mathrm{H}}^{*} X$ est $(d-1)$-connexe. L'intérêt de cette démarche est la simplification suivante : un module instable est $s$-nilpotent si et seulement si $\mathrm{T}^{n} M$ est $(s-1)$-connexe pour tout $n$ (voir la section 6 ). En vertu du scindement $\mathrm{T} M=M \oplus \overline{\mathrm{T}} M$, il apparaît que $M$ est $s$-nilpotent si et seulement si $M$ est $(s-1)$-connexe et $\overline{\mathrm{T}}^{n} M$ est $(s-1)$-connexe pour tout $n$. En particulier si $M$ est dans $\mathcal{U}_{1}, M$ est $s$-nilpotent si et seulement si $M$ et $\overline{\mathrm{T}} M$ sont $(s-1)$-connexes.

Il résulte de toutes les considérations précédentes qu'on peut supposer que $\overline{\mathrm{H}}^{*} X$ est dans $\mathcal{U}_{1}$ mais n'est pas localement finie, est $(d-1)$-connexe, est $d$-nilpotente et possède une structure d'algèbre triviale. 
Soit $\mathrm{F}(1)$ le sous-module instable de $\mathrm{H}^{*} \mathrm{~B} Z / 2$ engendré par la classe de degré un. La construction des classes $\alpha_{i, d}$ annoncée dans l'introduction repose la proposition suivante.

Proposition 7.2 Soit $M$ un module qui est dans $\mathcal{U}_{1}$ mais n'est pas localement fini. Soit $\eta$ l'unité d'adjonction $M \rightarrow \overline{\mathrm{T}} M \otimes \overline{\mathrm{H}}^{*} \mathrm{~B} \mathbb{Z} / 2$. Alors $\eta$ factorise par le sous-module $\overline{\mathrm{T}} M \otimes \mathrm{F}(1)$. De plus le noyau et le conoyau de

$$
\eta: M \rightarrow \overline{\mathrm{T}} M \otimes \mathrm{F}(1)
$$

sont localement finis.

Démonstration Dans [Sc2], ce résultat est démontré sous l'hypothèse que $M$ est de type fini. Tout module instable est colimite filtrante de ses sous-modules de type fini. Etant donné que les colimites filtrantes sont exactes, commutent aux produits tensoriels ainsi qu'au foncteur $\overline{\mathrm{T}}$, et du fait que $\mathcal{U}_{0}$ est stable par colimites, il vient que ce résultat est vrai en l'absence de toute hypothèse de finitude.

Lemme 7.3 Il existe un entier $i_{d}$ tel que pour tout $i \geq i_{d}$, il existe des classes $\alpha_{i, d}$ dans $\overline{\mathrm{H}}^{*} X$ qui vérifient:

- la classe $\alpha_{i, d}$ est de degré $2^{i}+d$,

- la classe $\alpha_{i, d}$ est exactement $d$-nilpotente,

- $\mathrm{Sq}^{2^{i}} \alpha_{i, d}=\alpha_{i+1, d}$.

Démonstration Posons $M=\overline{\mathrm{H}}^{*} X$. On applique la proposition 7.2. et on obtient une suite exacte :

$$
0 \longrightarrow L \longrightarrow M \stackrel{\eta}{\longrightarrow} \overline{\mathrm{T}} M \otimes \mathrm{F}(1) \longrightarrow L^{\prime} \longrightarrow 0
$$

où les modules instables $L$ et $L^{\prime}$ sont localement finis. Comme $\overline{\mathrm{T}} M$ est $(d-1)$ connexe, il existe $\alpha \in \overline{\mathrm{T}} M$ non nul et de degré $d$.

Etant donné que $\overline{\mathrm{T}} M$ est localement fini, il existe une borne entière $h$ telle que toute opération de Steenrod de degré plus grand que $h$ annule l'élément $\alpha$ de $\overline{\mathrm{T}} M$, i.e. $\mathcal{A}_{2}^{\geq h} . \alpha=0$.

Par conséquent, pour tout entier $i$ tel que $2^{i} \geq 2 h$, on a par la formule de Cartan,

$$
\mathrm{Sq}^{2^{i}} \alpha \otimes u^{2^{i}}=\alpha \otimes u^{2^{i+1}} .
$$

Soit $\kappa$ un entier tel que $2^{\kappa} \geq 2 h$. 
Il suit de (1) et du fait que $L^{\prime}$ est localement fini qu'il existe une borne $\kappa^{\prime} \geq \kappa$, telle que les classes $\alpha \otimes u^{2^{i}}$ de $\overline{\mathrm{T}} M \otimes \mathrm{F}(1)$ sont d'image nulle dans $L^{\prime}$ pour $i>\kappa^{\prime}$. Ainsi, l'élément $\alpha \otimes u^{2^{i}}$ provient pour $i \geq \kappa^{\prime}$ d'un élément $\alpha_{i, d}^{\prime}$ de $M$, i.e. $\eta\left(\alpha_{i, d}^{\prime}\right)=\alpha \otimes u^{2^{i}}$.

On choisit alors :

(1) $i_{d}=\kappa^{\prime}$,

(2) $\alpha_{i_{d}, d}=\alpha^{\prime} i_{d}, d$,

(3) pour tout $i \geq i_{d}, \alpha_{i+1, d}=\mathrm{Sq}^{2^{i}} \alpha_{i, d}$.

Ces classes vérifient le premier et le troisième point du lemme 7.3 par construction. Le second point découle du fait suivant : La formule de Cartan, l'action des carrés de Steenrod $\mathrm{Sq}^{i}$ sur $\mathrm{F}(1)$ et le fait que $2^{\kappa^{\prime}} \geq 2^{\kappa} \geq 2 h$ entraînent

$$
\forall c \geq 0, \forall t<d, \eta\left(\mathrm{Sq}_{t}^{c} \alpha_{i, d}\right)=\mathrm{Sq}_{t}^{c}\left(\alpha \otimes u^{2^{i}}\right)=\left(\mathrm{Sq}_{t}^{c} \alpha\right) \otimes u^{2^{i}} .
$$

Or le degré de $\mathrm{Sq}_{t}^{c} \alpha$ est une fonction strictement croissante de $c$ car $t<d$. Donc, pour $c$ suffisamment grand, $\eta\left(\mathrm{Sq}_{t}^{c} \alpha_{i, d}\right)=0$ car $\overline{\mathrm{T}} M$ est localement fini. De ce fait $\mathrm{Sq}_{t}^{c} \alpha_{i, d}$ provient de $L$ qui est localement fini. Donc à nouveau $\mathrm{Sq}_{t}^{c} \alpha_{i, d}$ est nul pour $c$ suffisamment grand, c'est-à-dire $\alpha_{i, d}$ est $t$-nilpotent.

Pour $t=d$, on a

$$
\eta\left(\mathrm{Sq}_{d} \alpha_{i, d}\right)=\mathrm{Sq}_{d}\left(\alpha \otimes u^{2^{i}}\right)=\alpha \otimes u^{2^{i+1}} \neq 0
$$

et donc pour tout entier $c$,

$$
\eta\left(\mathrm{Sq}_{d}^{c} \alpha_{i, d}\right)=\mathrm{Sq}_{d}^{c}\left(\alpha \otimes u^{2^{i}}\right)=\alpha \otimes u^{2^{i+c}} \neq 0
$$

ce qui montre que $\mathrm{Sq}_{d}^{c} \alpha_{i, d}$ est non nul quelque soit $c$. On a montré que $\alpha_{i, d}$ est exactement $d$-nilpotente, ce qui démontre le deuxième point du lemme.

\subsection{Construction des classes $\alpha_{i, \ell}$}

Pour $0 \leq \ell \leq d$, et pour tout $i \geq i_{d}$, on définit des classes $\alpha_{i, \ell}$ dans la cohomologie de $\Omega^{d-\ell} X$ de la manière suivante :

- les classes $\alpha_{i, d}$ sont définies par le lemme 7.3,

- la classe $\alpha_{i, \ell-1}$ est la désuspension de l'image de $\alpha_{i, \ell}$ par l'homorphisme de coin $\overline{\mathrm{H}}^{*} \Omega^{d-\ell} X \longrightarrow \Sigma \overline{\mathrm{H}}^{*} \Omega^{d-\ell+1} X$.

Lemme 7.4 Les classes $\alpha_{i, \ell}$ construites précédemment ont les propriétés suivantes, pour $d \geq \ell \geq 0$ et pour tout $i \geq i_{d}$ : 
(1) la classe $\alpha_{i, \ell}$ est de degré $2^{i}+\ell$,

(2) la classe $\alpha_{i, \ell}$ est exactement $\ell$-nilpotente,

(3) $\mathrm{Sq}^{2^{i}} \alpha_{i, \ell}=\alpha_{i+1, \ell}$,

(4) il existe un entier $i_{\ell}$ tel que pour tout $i \geq i_{\ell}$, le cup-carré $\alpha_{i, \ell} \cup \alpha_{i, \ell}$ est nul.

Démonstration Les trois premières affirmations du lemme sont faciles :

- le premier point résulte des définitions,

- le troisième point est conséquence des propriétés de compatibilité de la suite spectrale d'Eilenberg-Moore aux opérations de Steenrod,

- le deuxième point est conséquence du fait qu'on a des monomorphismes (voir la proposition A.4) qui sont induits par le morphisme de coin itéré :

$$
\mathrm{R}_{d} \overline{\mathrm{H}}^{*} X \hookrightarrow \mathrm{R}_{d-1} \overline{\mathrm{H}}^{*} \Omega X \hookrightarrow \ldots \hookrightarrow \mathrm{R}_{\ell} \overline{\mathrm{H}}^{*} \Omega^{d-\ell} X \hookrightarrow \ldots \hookrightarrow \mathrm{R}_{0} \overline{\mathrm{H}}^{*} \Omega^{d} X
$$

En effet, soit $\bar{\alpha}_{i, \ell}$ l'image de $\alpha_{i, \ell}$ dans $\mathrm{R}_{\ell} \overline{\mathrm{H}}^{*} \Omega^{d-\ell} X$. Les classes $\bar{\alpha}_{i, \ell}$ sont les images successives de $\bar{\alpha}_{i, d}$ par les monomorphismes précédents, et donc $\alpha_{i, \ell}$ est non nulle pour tout $\ell \leq d$. Une récurrence sur la proposition A.5 montre d'autre part que $\overline{\mathrm{H}}^{*} \Omega^{d-\ell} X$ est au moins $\ell$-nilpotent. Par conséquent les classes $\alpha_{i, \ell}$ sont au moins $\ell$-nilpotentes et réduisent non trivialement dans $\mathrm{R}_{\ell} \overline{\mathrm{H}}^{*} \Omega^{d-\ell} X$. C'est dire qu'elles sont exactement $\ell$ nilpotentes.

Le point 4 se démontre par une récurrence descendante, que l'on renvoie au prochain paragraphe.

Si l'on admet ce quatrième point, la démonstration de la conjecture de $\mathrm{N}$. Kuhn est terminée, car les deuxième et quatrième points du lemme 7.4 sont contradictoires pour $l=0$.

\subsection{Démonstration du point $4 \mathrm{du}$ lemme 7.4}

Remarquons tout d'abord que par application itérée des propositions A.6 et A.5 et du corollaire A.7, on a le lemme suivant :

Lemme 7.5 Pour $0 \leq \ell \leq d$, on a :

- Le module instable $\overline{\mathrm{H}}^{*} \Omega^{d-\ell} X$ est (au moins) $\ell$-nilpotent,

- le module instable $\mathrm{R}_{i} \mathrm{~F}_{-1} \overline{\mathrm{H}}^{*} \Omega^{d-\ell} X$ est dans $\mathcal{U}_{1}$ pour $i \leq 2 \ell$, 
- le module instable $\mathrm{R}_{i} \mathrm{~F}_{-2} \overline{\mathrm{H}}^{*} \Omega^{d-\ell} X$ est dans $\mathcal{U}_{2}$ pour $i \leq 2 \ell$,

- si $\ell \geq 1$ et si $i \leq 2 \ell-1$, le module $\mathrm{R}_{i} \mathrm{~F}_{-2} \overline{\mathrm{H}}^{*} \Omega^{d-\ell} X$ est en fait dans $\mathcal{U}_{1}$.

Pour démontrer le point $4 \mathrm{du}$ lemme 7.4 , on procède par récurrence descendante sur $\ell$.

Soit $\ell \geq 1$. Supposons que pour $i \geq i_{\ell}, \alpha_{i, \ell} \cup \alpha_{i, \ell}=0$.

On considère la suite spectrale d'Eilenberg-Moore qui relie la cohomologie de $\Omega^{d-\ell} X$ à celle de $\Omega^{d-\ell+1} X$.

Alors $\alpha_{i, \ell} \otimes \alpha_{i, \ell}$ définit un 1-cycle, qui est un cycle permanent. En effet, $\alpha_{i, \ell} \otimes$ $\alpha_{i, \ell}$ est exactement $2 \ell$-nilpotente et l'image des différentielles est constituée d'éléments de degré de nilpotence strictement supérieur à $2 \ell$. Soit $\omega_{i, \ell-1}$ une classe de $\mathrm{H}^{*} \Omega^{d-\ell+1} X$ qui est détectée par le cycle permanent induit par $\alpha_{i, \ell} \otimes$ $\alpha_{i, \ell}$.

Lemme 7.6 La classe $\omega_{i, \ell-1}$ est au moins $(2 \ell-2)$-nilpotente.

Démonstration Remarquons que cette condition est trivialement vérifiée si $\ell=1$.

Pour $\ell>1$, la classe $\omega_{i, \ell-1}$ est au moins $(\ell-1)$-nilpotente car le module $\mathrm{F}_{-2} \overline{\mathrm{H}}^{*} \Omega^{d-\ell+1}$ est $(\ell-1)$-nilpotent.

Soit ${\overline{\omega_{i, \ell-1}}}^{s}$ l'image de $\omega_{i, \ell-1}$ dans $\mathrm{R}_{s} \overline{\mathrm{H}}^{*} \Omega^{d-\ell+1} X$ pour $s \leq 2 \ell-3$. La classe ${\overline{\omega_{i, \ell-1}}}^{s}$ est de degré $2^{i+1}+2 \ell-2-s$. On a

$$
\alpha\left(2^{i+1}+2 \ell-2-s\right) \geq 2,
$$

or $\mathrm{R}_{s} \overline{\mathrm{H}}^{*} \mathrm{~F}_{-2} \Omega^{d-\ell+1} X$ est dans $\mathcal{U}_{1}$, pour $\ell-1 \leq s \leq 2 \ell-3$ d'après le lemme 7.5. De ce fait, pour $\ell-1 \leq s \leq 2 \ell-3,{\overline{\omega_{i, \ell-1}}}^{s}$ est nulle et donc $\omega_{i, \ell-1}$ est au moins $(2 \ell-2)$-nilpotente.

Lemme 7.7 On a l'égalité

$$
\mathrm{Sq}^{2^{i}} \omega_{i, \ell-1}=\alpha_{i, \ell-1} \cup \alpha_{i+1, \ell-1} \quad \bmod \left(\mathrm{F}_{-2} \overline{\mathrm{H}}^{*} \Omega^{d-\ell+1} X\right)_{2 \ell-2} .
$$

Démonstration On a par la formule de Cartan, pour $i \geq i_{\ell}$

$\mathrm{Sq}^{2^{i}}\left(\alpha_{i, \ell} \otimes \alpha_{i, \ell}\right)=\left(\mathrm{Sq}^{2^{i}} \alpha_{i, \ell}\right) \otimes \alpha_{i, \ell}+\alpha_{i, \ell} \otimes\left(\mathrm{Sq}^{2^{i}} \alpha_{i, \ell}\right)=\alpha_{i+1, \ell} \otimes \alpha_{i, \ell}+\alpha_{i, \ell} \otimes \alpha_{i+1, \ell}$.

Or $\alpha_{i+1, \ell} \otimes \alpha_{i, \ell}+\alpha_{i, \ell} \otimes \alpha_{i+1, \ell}$ est un cycle permanent qui n'est jamais l'image d'une différentielle (pour des raisons de nilpotence, comme précédemment) et qui détecte $\alpha_{i+1, \ell-1} \cup \alpha_{i, \ell-1}$. 
Ceci montre que la différence $z=\mathrm{Sq}^{2^{i}} \omega_{i, \ell-1}-\alpha_{i, \ell-1} \cup \alpha_{i+1, \ell-1}$ vit dans $\mathrm{F}_{-1} \overline{\mathrm{H}}^{*} \Omega^{d-\ell+1} X$.

On conclut en remarquant que ces classes sont de degré $2^{i}+2^{i+1}+2 \ell-2$. Or pour $s \leq 2 \ell-2$, on a $\alpha\left(2^{i}+2^{i+1}+2 \ell-2-s\right) \geq 2$. Comme le module $\mathrm{R}_{s} \mathrm{~F}_{-1} \overline{\mathrm{H}}^{*} \Omega^{d-\ell+1} X$ est dans $\mathcal{U}_{1}$ pour $s \leq 2 \ell-2$ d'après le lemme 7.5 , on en déduit l'égalité annoncée.

On déduit du lemme 7.7 qu'on a la relation

$$
\mathrm{Sq}^{2^{i}} \mathrm{Sq}^{2^{i}} \omega_{i, \ell-1}=\alpha_{i+1, \ell-1} \cup \alpha_{i+1, \ell-1} \quad \bmod \left(\mathrm{F}_{-2} \overline{\mathrm{H}}^{*} \Omega^{d-\ell+1} X\right)_{2 \ell-2} .
$$

Ceci résulte de la formule de Cartan, et du fait que $\mathrm{R}_{2 \ell-2} \mathrm{~F}_{-2} \overline{\mathrm{H}}^{*} \Omega^{d-\ell+1}$ est dans $\mathcal{U}_{2}$, par le lemme 7.5 .

On remarque alors que

(1) la relation $\mathrm{Sq}^{2^{i}} \mathrm{Sq}^{2^{i}} \omega_{i, \ell-1}=\alpha_{i+1, \ell-1} \cup \alpha_{i+1, \ell-1} \quad$ est vraie modulo des termes de degré de nilpotence strictement plus grand que $2 \ell-2$,

(2) l'image $\overline{\mathrm{Sq}^{2^{i}} \mathrm{Sq}^{2^{i}} \omega_{i, \ell-1}}$ de $\mathrm{Sq}^{2^{i}} \mathrm{Sq}^{2^{i}} \omega_{i, \ell-1}$ dans $\mathrm{R}_{2 \ell-2} \mathrm{~F}_{-2} \overline{\mathrm{H}}^{*} \Omega^{d-\ell+1} X$ est nulle.

Le deuxième point provient du résultat suivant [Sc3, lemme 5.7, p. 554] :

Lemme 7.8 Pour tout entier $n>0$

$$
\mathrm{Sq}^{2^{n}} \mathrm{Sq}^{2^{n}} \in \overline{\mathcal{A}}(n) \mathrm{Sq}^{2^{n}} \overline{\mathcal{A}}(n)
$$

où $\mathcal{A}(n)$ est la sous-algèbre engendrée par $\left\{\mathrm{Sq}^{2^{i}}, 0 \leq i \leq n-1\right\}$, et $\overline{\mathcal{A}}(n)$ est l'idéal des éléments strictement positifs de $\mathcal{A}(n)$.

Cette décomposition de l'opération de Steenrod $\mathrm{Sq}^{2^{i}} \mathrm{Sq}^{2^{i}}$ montre que $\overline{\mathrm{Sq}^{2^{i}} \mathrm{Sq}^{2^{i}} \omega_{i, \ell-1}}$ est de poids supérieur à 3 , alors que $\mathrm{R}_{2 \ell-2} \mathrm{H}^{*} \Omega^{d-\ell+1} X$ est dans $\mathcal{U}_{2}$, d'après le lemme 7.5.

Ceci montre que $\alpha_{i+1, \ell-1} \cup \alpha_{i+1, \ell-1}$ est au moins $(2 \ell-1)$-nilpotent et donc qu'il existe un entier $c$ tel que

$$
0=\operatorname{Sq}_{2 \ell-2}^{c}\left(\alpha_{i+1, \ell-1} \cup \alpha_{i+1, \ell-1}\right)=\alpha_{i+c+1, \ell-1} \cup \alpha_{i+c+1, \ell-1} .
$$

On peut donc choisir $i_{\ell+1}=i_{\ell}+c+1$, et on aura pour tout $i \geq i_{\ell+1}, \alpha_{i, \ell-1} \cup$ $\alpha_{i, \ell-1}=0$. 


\section{A Appendice}

\section{A.1 Filtration de Krull et filtration nilpotente}

Rappelons qu'on note pour $M$ un module instable et $s$ un entier $M_{s}$ le sousmodule instable de $M$ formé des éléments (au moins) $s$-nilpotents et $\mathrm{R}_{s} M$ le module $\Sigma^{-s}\left(M_{s} / M_{s+1}\right)$ (voir la section 6.1).

La proposition suivante affirme grosso modo que les foncteurs $\mathrm{R}_{s}$ sont exacts à gauche modulo $\mathcal{N}$ il.

Proposition A.1 Soit

$$
0 \longrightarrow A \longrightarrow B \longrightarrow C \longrightarrow 0
$$

une suite exacte dans $\mathcal{U}$ et soit $L$ le conoyau de $\mathrm{R}_{s} A \longrightarrow \mathrm{R}_{s} B$. Pour tout entier $s$, le morphisme $\mathrm{R}_{s} A \longrightarrow \mathrm{R}_{s} B$ est un monomorphisme et le noyau de $L \longrightarrow \mathrm{R}_{s} C$ est constitué d'éléments nilpotents.

Démonstration On chasse dans le diagramme commutatif suivant, dont les trois colonnes sont exactes.

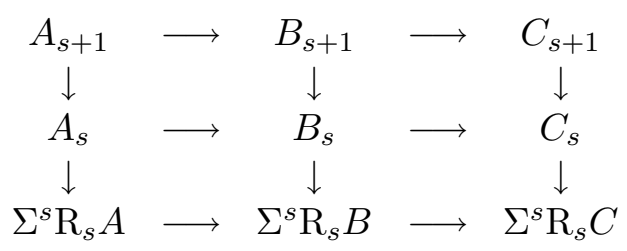

Le morphisme $\mathrm{R}_{s} A \longrightarrow \mathrm{R}_{s} B$ est un monomorphisme : Soit $x$ un élément de $\operatorname{Ker}\left(\mathrm{R}_{s} A \longrightarrow \mathrm{R}_{s} B\right)$. Soit $x_{1}$ un élément de $A_{s}$ qui se projette sur $\Sigma^{s} x \in$ $\Sigma^{s} \mathrm{R}_{s} A$.

Soit $x_{2}$ l'image de $x_{1}$ dans $B_{s}$. L'élément $x_{2}$ se projette sur 0 dans $\Sigma^{s} \mathrm{R}_{s} B$.

Par exactitude de la seconde colonne, l'élément $x_{2}$ est dans $B_{s+1}$, i.e. $x_{2}$ est $(s+1)$-nilpotent donc pour un certain entier $c$, on a $\left(\mathrm{Sq}_{s}\right)^{c} x_{2}=0$.

Donc par injectivité de $A_{s} \longrightarrow B_{s}$, il vient que $\left(\mathrm{Sq}_{s}\right)^{c} x_{1}=0$, i.e. l'élément $x_{1}$ est dans $A_{s+1}$ soit à dire que $x_{1}$ est $(s+1)$-nilpotent .

Par conséquent $x$ est nul, ce qui montre que $\mathrm{R}_{s} A \longrightarrow \mathrm{R}_{s} B$ est un monomorphisme.

Le noyau de $L \longrightarrow \mathrm{R}_{s} C$ est nilpotent : Soit $x$ un élément de $\operatorname{Ker}\left(\mathrm{R}_{s} B \longrightarrow \mathrm{R}_{s} C\right)$ et soit $x_{1}$ un relèvement de $\Sigma^{s} x$ dans $B_{s}$. Soit $x_{2}$ l'image de $x_{1}$ dans $C_{s}$. 
L'élément $x_{2}$ se projette sur 0 dans $\Sigma^{s} \mathrm{R}_{s} C$, donc $x_{2}$ est dans $C_{s+1}$, i.e. $x_{2}$ est $(s+1)$-nilpotent et pour un certain entier $c$, on a $\left(\mathrm{Sq}_{s}\right)^{c} x_{2}=0$.

Par conséquent, l'image de $\left(\mathrm{Sq}_{s}\right)^{c} x_{1}$ dans $C$ est nulle et par exactitude de la suite $A \longrightarrow B \longrightarrow C$, il existe un élément $x_{3}$ de $A$ dont l'image est $\left(\mathrm{Sq}_{s}\right)^{c} x_{1}$. Comme $\left(\mathrm{Sq}_{s}\right)^{c} x_{1}$ est au moins $s$-nilpotent et comme $A \rightarrow B$ est un monomorphisme, l'élément $x_{3}$ est au moins $s$-nilpotent.

Soit $\Sigma^{s} x_{4}$ l'image de $x_{3}$ dans $\Sigma^{s} \mathrm{R}_{s} A$. L'image de $\Sigma^{s} x_{4}$ dans $\Sigma^{s} \mathrm{R}_{s} B$ est $\left(\mathrm{Sq}_{s}\right)^{c} \Sigma^{s} x=\Sigma^{s}\left(\mathrm{Sq}_{0}\right)^{c} x$. Donc l'image de $x$ dans $L$ est nilpotent.

Corollaire A.2 Soient $0 \rightarrow A \rightarrow B \rightarrow C \rightarrow 0$ une suite exacte dans $\mathcal{U}$. On suppose que $A$ est $\ell$-nilpotent pour un entier $\ell \geq 1$; alors le morphisme $\mathrm{R}_{s} B \longrightarrow \mathrm{R}_{s} C$ est un isomorphisme pour tout entier $s<\ell$ (et un épimorphisme pour $s=\ell$ ).

Démonstration Le module $\mathrm{R}_{s} A$ est nul pour tout $s<\ell$. La proposition affirme alors que le noyau du morphisme $\mathrm{R}_{s} B \longrightarrow \mathrm{R}_{s} C$ est nilpotent pour tout $s<\ell$. Or $\mathrm{R}_{s} B$ est un module réduit, donc ce noyau est nul.

Pour achever la preuve, il suffit de montrer que le morphisme $B_{s} \rightarrow C_{s}$ est un épimorphisme pour tout $s \leq \ell$. Soit donc $s$ un tel entier et $x$ un élément $s$-nilpotent de $C$. Il provient d'un élément $x_{1}$ de $B$ et pour tout $s^{\prime}<s$ il existe un certain entier $c$ et un élément $x_{2}$ de $A$ tel que $\left(\mathrm{Sq}_{s^{\prime}}\right)^{c} x_{1}$ est l'image de $x_{2}$ par le morphisme $A \rightarrow B$. Or $A$ est $\ell$-nilpotent donc $\left(\mathrm{Sq}_{s^{\prime}}\right)^{c^{\prime}} x_{2}$ est nul pour $c^{\prime}$ assez grand. On en déduit que $x_{1}$ est $s$-nilpotent, c'est-à-dire $x_{1} \in B_{s}$.

Corollaire A.3 Soient $0 \rightarrow A \rightarrow B \rightarrow C \rightarrow 0$ une suite exacte de modules instables et $p, q, s$ trois entiers positifs. On suppose que $\mathrm{R}_{s} A$ est dans $\mathcal{U}_{p}$ et que $\mathrm{R}_{s} C$ est dans $\mathcal{U}_{q}$; alors $\mathrm{R}_{s} B$ est dans $\mathcal{U}_{\max \{p, q\}}$.

Démonstration Le foncteur $\mathrm{R}_{s}$ transforme la suite exacte

$$
0 \rightarrow A \rightarrow B \rightarrow C \rightarrow 0
$$

en une suite

$$
0 \rightarrow \mathrm{R}_{s} A \rightarrow \mathrm{R}_{s} B \rightarrow \mathrm{R}_{s} C
$$

exacte au sens de la proposition A.1.

Les modules $\mathrm{R}_{s} A, \mathrm{R}_{s} B$ et $\mathrm{R}_{s} C$ sont réduits, ce qui nous autorise à appliquer la caractérisation 6.2 de la filtration de Krull en terme de filtration par le poids pour les modules réduits. Il suffit donc de montrer que $\mathrm{R}_{s} B$ est de poids inférieur ou égal à $\max \{p, q\}$, i.e. que $\mathrm{R}_{s} B$ est nul dans les degrés $i$ tels que 
$\alpha(i)>\max \{p, q\}$. Rappelons que $\alpha(i)$ est le nombre de puissances de deux composant l'écriture diadique de $i$.

Soit $x$ un élément de $\mathrm{R}_{s} B$ en degré $|x|$ tel que $\alpha(|x|)>\max \{p, q\}$. L'image de $x$ par $\mathrm{R}_{s} B \rightarrow \mathrm{R}_{s} C$ est nulle car $\mathrm{R}_{s} C$ est dans $\mathcal{U}_{q}$ et donc de poids inférieur ou égal à $q \leq \max \{p, q\}$. D'après la proposition A.1, il existe un entier $c$ tel que $S q_{0}^{c} x$ est dans $\mathrm{R}_{s} A$. Le degré de $S q_{0}^{c} x$ est $2^{c}|x|$ et $\alpha\left(2^{c}|x|\right)=\alpha(|x|)>p$. Or $\mathrm{R}_{s} A$ est dans $\mathcal{U}_{p}$, donc de poids inférieur ou égal à $p$. Il s'ensuit que $S q_{0}^{c} x$ est nul, et donc $x$ est nul car $\mathrm{R}_{s} A$ est réduit.

\section{A.2 Suite spectrale d'Eilenberg-Moore et filtration nilpotente}

Dans toute cette section, $X$ est un espace profini pointé. On va dégager suivant [Sc3] quelques propriétés de compatibilité entre la filtration nilpotente de la cohomologie continue réduite de $X$ et celle de son espace de lacets $\Omega X$ en utilisant la spectrale d'Eilenberg-Moore.

Le terme $\mathrm{E}_{r}^{-s, *}$ de cette suite spectrale est naturellement muni d'une structure de module instable pour tous entiers $s$ et $r \geq 1$. De plus, la différentielle $\mathrm{d}_{r}$ est un morphisme de modules instables

$$
\mathrm{d}_{r}: \mathrm{E}_{r}^{-s-r, *} \longrightarrow \Sigma^{r-1} \mathrm{E}_{r}^{-s, *} .
$$

Enfin, la cohomologie continue réduite de $\Omega X$ est naturellement munie d'une filtration croissante

$$
0=\mathrm{F}_{0} \overline{\mathrm{H}}^{*} \Omega X \subset \mathrm{F}_{-1} \overline{\mathrm{H}}^{*} \Omega X \subset \mathrm{F}_{-2} \overline{\mathrm{H}}^{*} \Omega X \subset \ldots \subset \mathrm{F}_{-s} \overline{\mathrm{H}}^{*} \Omega X \subset \ldots \subset \overline{\mathrm{H}}^{*} \Omega X
$$

par des sous-modules instables telle qu'on a pour tout $s \geq 1$ un isomorphisme

$$
\mathrm{E}_{\infty}^{-s, *} \cong \Sigma^{s}\left(\mathrm{~F}_{-s} \overline{\mathrm{H}}^{*} \Omega X / \mathrm{F}_{-s+1} \overline{\mathrm{H}}^{*} \Omega X\right) \text {. }
$$

Cette filtration est convergente :

$$
\bigcup_{i \in \mathbb{N}} \mathrm{F}_{-i} \overline{\mathrm{H}}^{*} \Omega X=\overline{\mathrm{H}}^{*} \Omega X
$$

(Voir la section 4.)

Proposition A.4 Soit $X$ un espace profini pointé dont la cohomologie réduite est dans $\mathcal{N} i l_{\ell}$ pour un entier $\ell \geq 1$. L'homomorphisme de coin induit pour $s \leq 2 \ell-1$ un monomorphisme

$$
\mathrm{R}_{s} \overline{\mathrm{H}}^{*} X \hookrightarrow \mathrm{R}_{s-1} \overline{\mathrm{H}}^{*} \Omega X .
$$


Démonstration L'homomorphisme de coin $\overline{\mathrm{H}}^{*} X \longrightarrow \Sigma \overline{\mathrm{H}}^{*} \Omega X$ factorise de la manière suivante:

$$
\overline{\mathrm{H}}^{*} X \cong \mathrm{E}_{1}^{-1, *} \rightarrow \mathrm{E}_{\infty}^{-1, *} \cong \Sigma \mathrm{F}_{-1} \overline{\mathrm{H}}^{*} \Omega X \hookrightarrow \Sigma \overline{\mathrm{H}}^{*} \Omega X
$$

Le morphisme $\mathrm{E}_{1}^{-1, *} \longrightarrow \mathrm{E}_{\infty}^{-1, *}$ est la colimite sur $r$ des morphismes $\mathrm{E}_{1}^{-1, *} \longrightarrow$ $\mathrm{E}_{r}^{-1, *}$, lesquels sont les composés $\mathrm{E}_{1}^{-1, *} \rightarrow \mathrm{E}_{2}^{-1, *} \rightarrow \cdots \rightarrow \mathrm{E}_{r-1}^{-1, *} \rightarrow \mathrm{E}_{r}^{-1, *}$.

Chaque morphisme $\mathrm{E}_{r}^{-1, *} \longrightarrow \mathrm{E}_{r+1}^{-1, *}$ est le conoyau de la différentielle $\mathrm{d}_{r}$ : $\Sigma^{1-r} \mathrm{E}_{r}^{-1-r, *} \longrightarrow \mathrm{E}_{r}^{-1, *}$. Comme le module $\mathrm{E}_{r}^{-1-r, *}$ est un sous-quotient de $\left(\overline{\mathrm{H}}^{*} X\right)^{\otimes(r+1)}$ qui est $(r+1) \ell$-nilpotent, le noyau du morphisme $\mathrm{E}_{r}^{-1, *} \longrightarrow \mathrm{E}_{r+1}^{-1, *}$ est formé d'éléments au moins $((r+1) \ell-(r-1))$-nilpotents donc au moins $2 \ell$-nilpotents puisqu'on a $r \geq 1$ et $\ell \geq 1$.

Le corollaire A.2 montre alors que le morphisme $\mathrm{R}_{s} \mathrm{E}_{r}^{-1, *} \longrightarrow \mathrm{R}_{s} \mathrm{E}_{r+1}^{-1, *}$ est un isomorphisme pour tout $s<2 \ell$. Par conséquent, le morphisme $\mathrm{R}_{s} \mathrm{E}_{1}^{-1, *} \longrightarrow$ $\mathrm{R}_{s} \mathrm{E}_{\infty}^{-1, *}$ est un isomorphisme pour $s<2 \ell$.

On a donc un isomorphisme $\mathrm{R}_{s} \overline{\mathrm{H}}^{*} X \longrightarrow \mathrm{R}_{s-1} \mathrm{~F}_{-1} \overline{\mathrm{H}}^{*} \Omega X$ qui se compose avec le monomorphisme $\mathrm{R}_{s-1} \mathrm{~F}_{-1} \overline{\mathrm{H}}^{*} \Omega X \longrightarrow \mathrm{R}_{s-1} \overline{\mathrm{H}}^{*} \Omega X$ (le foncteur $\mathrm{R}_{s-1}$ préserve les monomorphismes d'après la proposition A.1) en le monomorphisme souhaité.

Proposition A.5 Soient $X$ un espace profini pointé et $\ell \geq 1$ un entier tels que :

- la cohomologie réduite de $X$ est $\ell$-nilpotente,

- $\overline{\mathrm{H}}^{*} X /\left(\overline{\mathrm{H}}^{*} X\right)_{2 \ell}$ est dans $\mathcal{U}_{1}$.

Alors :

(1) la cohomologie réduite de $\Omega X$ est $(\ell-1)$-nilpotente,

(2) pour $s \leq 2 \ell-2$, le module instable $\mathrm{R}_{s} \mathrm{~F}_{-1} \overline{\mathrm{H}}^{*} \Omega X$ est dans $\mathcal{U}_{1}$,

(3) pour $s \leq 2 \ell-2$, le module instable $\mathrm{R}_{s} \mathrm{~F}_{-2} \overline{\mathrm{H}}^{*} \Omega X$ est dans $\mathcal{U}_{2}$.

Démonstration du point 1 Pour tout entier $s$, le terme $\mathrm{E}_{\infty}^{-s, *}$ est un sousquotient de $\left(\overline{\mathrm{H}}^{*} X\right)^{\otimes s}$ qui est $s \ell$-nilpotent. Donc pour tout entier $s$, le module $\mathrm{F}_{-s} \overline{\mathrm{H}}^{*} \Omega X / \mathrm{F}_{-s+1} \overline{\mathrm{H}}^{*} \Omega X=\Sigma^{-s} \mathrm{E}_{\infty}^{-s, *}$ est $(s \ell-s)$-nilpotent. On en déduit par récurrence, en utilisant que $\mathcal{N} i l_{\ell-1}$ est de Serre, que pour tout entier $s$, le module instable $\mathrm{F}_{-s} \overline{\mathrm{H}}^{*} \Omega X$ est $(\ell-1)$-nilpotent. Par suite, comme la suite spectrale est convergente et $\mathcal{N} i l_{\ell-1}$ stable par colimite, le module $\overline{\mathrm{H}}^{*} \Omega X$ est aussi $(\ell-1)$-nilpotent. 
Démonstration du point 2 Le module $\mathrm{E}_{\infty}^{-1, *}=\Sigma \mathrm{F}_{-1} \overline{\mathrm{H}}^{*} \Omega X$ est un quotient de $\mathrm{H}^{*} X$ par des éléments au moins $2 \ell$-nilpotents. On a donc un épimorphisme

$$
\mathrm{E}_{\infty}^{-1, *} \rightarrow \overline{\mathrm{H}}^{*} X /\left(\overline{\mathrm{H}}^{*} X\right)_{2 \ell}
$$

de noyau au moins $2 \ell$-nilpotent. En vertu du corollaire A.2, on a pour $s \leq 2 \ell-1$ un monomorphisme $\mathrm{R}_{s} \mathrm{E}_{\infty}^{-1, *} \longrightarrow \mathrm{R}_{s}\left(\overline{\mathrm{H}}^{*} X /\left(\overline{\mathrm{H}}^{*} X\right)_{2 \ell}\right)$. Or $\overline{\mathrm{H}}^{*} X /\left(\overline{\mathrm{H}}^{*} X\right)_{2 \ell}$ est dans $\mathcal{U}_{1}$ par hypothèse et donc aussi $\mathrm{R}_{s}\left(\overline{\mathrm{H}}^{*} X /\left(\overline{\mathrm{H}}^{*} X\right)_{2 \ell}\right)$ (car $\mathcal{U}_{1}$ est de Serre). Il suit que $\mathrm{R}_{s} \mathrm{E}_{\infty}^{-1, *}$ est également dans $\mathcal{U}_{1}$.

On obtient que

$$
\mathrm{R}_{s} \mathrm{E}_{\infty}^{-1, *} \cong \mathrm{R}_{s-1} \Sigma^{-1} \mathrm{E}_{\infty}^{-1, *} \cong \mathrm{R}_{s-1} \mathrm{~F}_{-1} \overline{\mathrm{H}}^{*} \Omega X
$$

est dans $\mathcal{U}_{1}$ pour tout $s \leq 2 \ell-2$, ce qui montre le second point de la proposition.

Démonstration du point 3 La suite exacte

$$
0 \longrightarrow \mathrm{F}_{-1} \overline{\mathrm{H}}^{*} \Omega X \longrightarrow \mathrm{F}_{-2} \overline{\mathrm{H}}^{*} \Omega X \longrightarrow \Sigma^{-2} \mathrm{E}_{\infty}^{-2, *} \longrightarrow 0
$$

et le corollaire A.3 assurent que pour montrer le troisième point du lemme, il suffit de montrer que pour tout $s \leq 2 \ell-2$, les modules $\mathrm{R}_{s} \mathrm{~F}_{-1} \overline{\mathrm{H}}^{*} \Omega X$ et $\mathrm{R}_{s} \Sigma^{-2} \mathrm{E}_{\infty}^{-2, *}$ sont dans $\mathcal{U}_{2}$.

Le fait que $\mathrm{R}_{s} \mathrm{~F}_{-1} \overline{\mathrm{H}}^{*} \Omega X$ est dans $\mathcal{U}_{2}$ pour $s \leq 2 \ell-2$ est conséquence du point 2 de cette proposition, car $\mathcal{U}_{1}$ est une sous-catégorie de $\mathcal{U}_{2}$.

Il reste donc à montrer que $\mathrm{R}_{s} \Sigma^{-2} \mathrm{E}_{\infty}^{-2, *}$ est dans $\mathcal{U}_{2}$. Le module instable $\mathrm{E}_{\infty}^{-2, *}$ est un sous-quotient de $\overline{\mathrm{H}}^{*} X^{\otimes 2}$, i.e. $\mathrm{E}_{\infty}^{-2, *}$ s'écrit $C / B$, avec $C \subset \overline{\mathrm{H}}^{*} X^{\otimes 2}$. De plus, le module $B$ est au moins $3 \ell$-nilpotent pour la raison suivante. On a

$$
\mathrm{d}_{r}: \mathrm{E}_{r}^{-2-r, *} \longrightarrow \Sigma^{(r-1)} \mathrm{E}_{r}^{-2, *},
$$

ce qui montre que $B$ est constitué d'éléments de degré de nilpotence au moins égal à $(r+2) \ell-(r-1) \geq 3 \ell$. On en déduit avec le corollaire A.2 que pour $s<3 \ell$, on a des monomorphismes:

$$
\mathrm{R}_{s} \mathrm{E}_{\infty}^{-2, *} \hookrightarrow \mathrm{R}_{s} C \hookrightarrow \mathrm{R}_{s}\left(\overline{\mathrm{H}}^{*} X^{\otimes 2}\right) .
$$

Le module $\left(\overline{\mathrm{H}}^{*} X\right)^{\otimes 2}$ est $2 \ell$-nilpotent par hypothèse, et donc le plus petit $s$ tel que $\mathrm{R}_{s}\left(\overline{\mathrm{H}}^{*} X^{\otimes 2}\right)$ est non trivial est $s=2 \ell$. Or $\mathrm{R}_{2 \ell}\left(\overline{\mathrm{H}}^{*} X^{\otimes 2}\right)$ est isomorphe à $\left(\mathrm{R}_{\ell} \overline{\mathrm{H}}^{*} X\right)^{\otimes 2}$ qui est dans $\mathcal{U}_{2}$ (voir la partie 6). Donc $\mathrm{R}_{2 \ell} \mathrm{E}_{\infty}^{-2, *}$ est dans $\mathcal{U}_{2}$. Finalement, $\mathrm{R}_{2 \ell-2} \Sigma^{-2} \mathrm{E}_{\infty}^{-2, *} \cong \mathrm{R}_{2 \ell} \mathrm{E}_{\infty}^{-2, *}$ est dans $\mathcal{U}_{2}$.

Proposition A.6 Soit $\ell>1$ un entier et soit $X$ un espace profini pointé. Si $\overline{\mathrm{H}}^{*} X$ est $\ell$-nilpotent et si $\overline{\mathrm{H}}^{*} X /\left(\overline{\mathrm{H}}^{*} X\right)_{2 \ell}$ est dans $\mathcal{U}_{1}$, alors $\overline{\mathrm{H}}^{*} \Omega X /\left(\overline{\mathrm{H}}^{*} \Omega X\right)_{2(\ell-1)}$ est également dans $\mathcal{U}_{1}$. 
Démonstration Le module instable $\mathrm{E}_{\infty}^{-s, *}$ est un sous-quotient de $\overline{\mathrm{H}}^{*} X^{\otimes s}$, donc $\mathrm{E}_{\infty}^{-s, *}$ est $s \ell$-nilpotent et $\mathrm{F}_{-s} \overline{\mathrm{H}}^{*} \Omega X / \mathrm{F}_{-s+1} \overline{\mathrm{H}}^{*} \Omega X=\Sigma^{-s} \mathrm{E}_{\infty}^{-s, *}$ est $s(\ell-1)$ nilpotent. On montre par récurrence sur $s$ que $\mathrm{F}_{-s} \overline{\mathrm{H}}^{*} \Omega X / \mathrm{F}_{-1} \overline{\mathrm{H}}^{*} \Omega X$ est $2(\ell-$ 1)-nilpotent. Par convergence de la suite spectrale, le module $\overline{\mathrm{H}}^{*} \Omega X / \mathrm{F}_{-1} \overline{\mathrm{H}}^{*} \Omega X$ est aussi $2(\ell-1)$-nilpotent.

On a une suite exacte:

$$
0 \longrightarrow \mathrm{F}_{-1} \overline{\mathrm{H}}^{*} \Omega X \longrightarrow \overline{\mathrm{H}}^{*} \Omega X \longrightarrow \overline{\mathrm{H}}^{*} \Omega X / \mathrm{F}_{-1} \overline{\mathrm{H}}^{*} \Omega X \longrightarrow 0
$$

dont le dernier terme $\overline{\mathrm{H}}^{*} \Omega X / \mathrm{F}_{-1} \overline{\mathrm{H}}^{*} \Omega X$ est $2(\ell-1)$-nilpotent. En particulier, pour $s \leq 2 \ell-3$, le module $\mathrm{R}_{s}\left(\overline{\mathrm{H}}^{*} \Omega X / \mathrm{F}_{-1} \overline{\mathrm{H}}^{*} \Omega X\right)$ est nul et donc dans $\mathcal{U}_{0}$. De plus, d'après la proposition précédente, pour tout $s \leq 2 \ell-3$, le module $\mathrm{R}_{s} \mathrm{~F}_{-1} \overline{\mathrm{H}}^{*} \Omega X$ est dans $\mathcal{U}_{1}$.

Le corollaire A.3 assure que pour tout $s \leq 2 \ell-3$, le module $\mathrm{R}_{s} \overline{\mathrm{H}}^{*} \Omega X$ est

dans $\mathcal{U}_{1}$. Comme $\mathcal{U}_{1}$ est de Serre, on obtient que $\overline{\mathrm{H}}^{*} \Omega X /\left(\overline{\mathrm{H}}^{*} \Omega X\right)_{2 \ell-2}$ est dans $\mathcal{U}_{1}$.

Corollaire A.7 Soit $\ell>1$ un entier et soit $X$ un espace profini pointé. Si $\overline{\mathrm{H}}^{*} X$ est $\ell$-nilpotent et si $\overline{\mathrm{H}}^{*} X /\left(\overline{\mathrm{H}}^{*} X\right)_{2 \ell}$ est dans $\mathcal{U}_{1}$, alors pour $s \leq 2 \ell-3$, le module $\mathrm{R}_{s} \mathrm{~F}_{-2} \overline{\mathrm{H}}^{*} \Omega X$ est dans $\mathcal{U}_{1}$.

Démonstration Le module $\mathrm{R}_{s} \mathrm{~F}_{-2} \overline{\mathrm{H}}^{*} \Omega X$ est un sous-module de $\mathrm{R}_{s} \overline{\mathrm{H}}^{*} \Omega X$ d'après la proposition A.1. Or pour $s \leq 2 \ell-3$, le module $\mathrm{R}_{s} \overline{\mathrm{H}}^{*} \Omega X$ est isomorphe à $\mathrm{R}_{s}\left(\overline{\mathrm{H}}^{*} \Omega X /\left(\overline{\mathrm{H}}^{*} \Omega X\right)_{2 \ell-2}\right)$, d'après le corollaire A.2. $O r \mathrm{R}_{s}\left(\overline{\mathrm{H}}^{*} \Omega X /\left(\overline{\mathrm{H}}^{*} \Omega X\right)_{2 \ell-2}\right)$ est dans $\mathcal{U}_{1}$ comme sous-quotient de $\overline{\mathrm{H}}^{*} \Omega X /\left(\overline{\mathrm{H}}^{*} \Omega X\right)_{2 \ell-2}$ qui d'après la proposition A.6 est dans $\mathcal{U}_{1}$.

\section{Références}

[Ad] J. F. Adams, On the non-existence of elements of Hopf invariant one. Ann. of Math. (2) 72 (1960), 20-104.

[AM] M. Artin, B. Mazur, Etale Homotopy, Springer L. N. M., 100, 1969.

[Boa] J. M. Boardman, Conditionally Convergent Spectral Sequences, Contemp. Math. 239 (1999), 49-84.

[Bou] A. K. Bousfield, On the homology spectral sequence of a cosimplicial space, Amer. J. Math., 109 (1987), 361-394.

[BK] A. K. Bousfield, D. M. Kan, Homotopy Limits, Completions, and Localizations, Springer L. N. M., 304, 1972.

[Do] R. et A. Douady, Théories galoisiennes 1, Cedic/Fernand Nathan, 1979. 
[DrS] E. Dror Farjoun, J. Smith, A Geometric Interpretation of Lannes' Functor T, Théorie de l'Homotopie, Astérisque, 191 (1990), 87-95.

[Dw] W.G. Dwyer, Strong convergence of the Eilenberg-Moore spectral sequence, Topology, 13 (1974), 255-265.

[DwS] W.G. Dwyer, J. Splalinski, Homotopy Theories and Model Categories, Handbook of algebraic topology, 1995, 73-126.

[FS] V. Franjou, L. Schwartz, Reduced unstable $\mathcal{A}$-modules and the modular representation theory of the symmetric groups, Ann. Sci. Ec. Norm. Supér., 23, No 4 (1990), 593-624.

[Ga] P. Gabriel, Des catégories abéliennes, Bull. Soc. Math. France, 90 (1962), 323448.

[GJ] P.G. Goerss, J.F. Jardine, Simplicial Homotopy Theory, Progress in Mathematics, 174, 1999.

[Go] T.G. Goodwillie, A remark on the homology of cosimplicial spaces, J. Pure Appl. Algebra, 127 (1998), 167-175.

[HLS] H. W. Henn, J. Lannes, L. Schwartz, The categories of unstable modules and unstable algebras modulo nilpotent objects, Am. J. of Math., 115, No 5 (1993), 1053-1106.

[Ku] N. J. Kuhn, On topologicaly realizing modules over the Steenrod algebra, Annals of Mathematics, 141 (1995), 321-347.

[La] J. Lannes, Sur les espaces fonctionnels dont la source est le classifiant d'un p-groupe abélien élémentaire, Publ. Math. I. H. E. S., 75 (1992), 135-244.

[Mac] S. Mac Lane, Homology, Springer, 1975.

[May] P. May, Simplicial Objects in Algebraic Topology, Van Nostrand, 1967.

[Mo] F. Morel, Ensembles profinis simpliciaux et interprétation géométrique du foncteur T, Bull. Soc. Math. France, 124 (1996), 347-373.

[Q] D.G. Quillen, Homotopical Algebra, Springer L. N. M., 43, 1967.

[Q2] D.G. Quillen, An application of simplicial profinite groups, Comment. Math. Helv., 44 (1969), 45-60.

[R] D. Rector, Steenrod operations in the Eilenberg-Moore spectral sequence, Comment. Math. Helv., 45 (1970), 540-552.

[Sc1] L. Schwartz, Unstable Modules over the Steenrod Algebra and Sullivan's Fixed Point Set Conjecture, University of Chicago Press, 1994.

[Sc2] L. Schwartz, A propos de la conjecture de non réalisation due a N. Kuhn, Invent. Math., 134, No 1, 211-227 (1998).

[Sc3] L. Schwartz, La filtration de Krull de la catégorie $\mathcal{U}$ et la cohomologie des espaces, Algebraic and Geometric topology, 1, 519-548, (2001).

[Sh] B.E. Shipley, Convergence of the homology spectral sequence of a cosimplicial space, Am. J. Math., 118 (1996), 179-207.

[SE] N.E. Steenrod, D.B.A. Epstein, Cohomology operations, Princeton Univ. Press, 1962. 
Laboratoire J.A. Dieudonné, Université de Nice Sophia-Antipolis Parc Valrose - BP 2053 - 06101 Nice, France

and

Laboratoire Jean Leray (UMR 6629 du C.N.R.S.), Université de Nantes BP 92208 - 44322 Nantes Cedex 3, France

Email: dehon@math.unice.fr, gaudens@math.univ-nantes.fr

Received: 29 November 2002 Revised: 3 May 2003 